\title{
Branes, partition functions, and quadratic monopole superpotentials
}

\author{
Antonio Amariti, ${ }^{1, *}$ Luca Cassia, ${ }^{2, \dagger}$ Ivan Garozzo, ${ }^{3,4, \$}$ and Noppadol Mekareeya ${ }^{3,5,8}$ \\ ${ }^{1}$ INFN, Sezione di Milano, Via Celoria 16, I-20133 Milano, Italy \\ ${ }^{2}$ Department of Physics and Astronomy, Uppsala University, Box 516, SE-75120 Uppsala, Sweden \\ ${ }^{3}$ INFN, sezione di Milano-Bicocca, Piazza della Scienza 3, I-20126 Milano, Italy \\ ${ }^{4}$ Dipartimento di Fisica, Università di Milano-Bicocca, Piazza della Scienza 3, I-20126 Milano, Italy \\ ${ }^{5}$ Department of Physics, Faculty of Science, Chulalongkorn University, \\ Phayathai Road, Pathumwan, Bangkok 10330, Thailand
}

(Received 21 May 2019; published 1 August 2019)

\begin{abstract}
We obtain the brane setup describing $3 \mathrm{~d} \mathcal{N}=2$ dualities for $U \operatorname{Sp}\left(2 N_{c}\right)$ and $U\left(N_{c}\right)$ SQCD with monopole superpotentials. This classification follows from a complete analysis of affine and twisted affine compactifications from $4 \mathrm{~d}$. The analysis leads to a new duality for the unitary case that has previously been overlooked in the literature. We check this by matching the three-sphere partition function of the two sides of this new duality and find a perfect agreement. Furthermore, we use the partition function to predict new $3 \mathrm{~d} \mathcal{N}=2$ dualities for SQCD with monopole superpotentials and tensorial matter.
\end{abstract}

DOI: 10.1103/PhysRevD.100.046001

\section{INTRODUCTION}

In the recent past there has been remarkable progress in the understanding of $3 \mathrm{~d}$ dualities with and without supersymmetry. One of the main roles in the discovery of new dualities and in the appearance of new phenomena such as symmetry enhancements has been played by monopole operators. The reason is that these operators can be used to modify the path integral and to constrain the global symmetries. These constraints give raise to nontrivial IR relations, deforming old dualities and generating new ones. For example, this phenomenon has been largely studied in $3 \mathrm{~d} \mathcal{N}=2 \mathrm{SQCD}$ with linear and quadratic monopole superpotentials.

These constructions have led to a series of new results in the last decade. For example, linear monopole superpotentials were used in [1] to explain how to reduce 4d Seiberg duality to $3 \mathrm{~d}$ (see also [2] for an earlier attempt). This construction was then generalized to theories with more sophisticated gauge and field content in [3-7]. Moreover, the string theory interpretation of this reduction was obtained in [8-11], by engineering the linear monopole superpotential in terms of D1 branes, along the lines of the construction of [12-15].

\footnotetext{
*antonio.amariti@mi.infn.it

†luca.cassia@physics.uu.se

*ivangarozzo@gmail.com

§n.mekareeya@gmail.com
}

Published by the American Physical Society under the terms of the Creative Commons Attribution 4.0 International license. Further distribution of this work must maintain attribution to the author(s) and the published article's title, journal citation, and DOI. Funded by SCOAP.
A similar construction was provided in [16] to explain the dimensional reduction of $4 \mathrm{~d} \mathcal{N}=1 S U(2) \mathrm{SQCD}$ with eight fundamentals. The presence of a monopole superpotential was crucial in explaining the enhancement of the $S U(8)$ global symmetry to $E_{7}$. By real mass flow it was then shown that there are more general types of monopole superpotentials for $U(1)$ theories. The generalization of this phenomenon to $\operatorname{USp}\left(2 N_{c}\right)$ with an antisymmetric and eight fundamentals was recently discussed in [17-19]. The $U\left(N_{c}\right)$ generalization of the superpotentials introduced in [16] for the $U(1)$ models was obtained in [20,21]. This construction was then used in [22-25] to dimensionally reduce the $4 \mathrm{~d} \mathcal{N}=1$ "Argyres-Douglas Lagrangians" discovered in [26-30]. Moreover, monopole superpotentials have allowed the physical interpretation of many mathematical identities among hyperbolic hypergeometric integrals [31]. Such identities indeed represent the matching of the three-sphere partition function between models with monopole superpotentials turned on. Other interesting results involving monopole superpotentials have been discussed in [32-36].

Furthermore, some other dualities, originally conjectured in [21], involve deformations with quadratic monopole operators. These dualities have been studied extensively in [37], also for the case of real gauge groups. In this paper we further investigate such dualities, providing two main results:

(i) We provide the D-brane engineering of the dualities discussed in $[21,37]$ involving quadratic monopole superpotentials. As a bonus we obtain a new duality previously overlooked in the literature.

(ii) We find new dualities with quadratic monopole superpotentials for $U\left(N_{c}\right)$ SQCD with and adjoint and $\operatorname{USp}\left(2 N_{c}\right)$ SQCD with an antisymmetric. 


\section{A. D-brane engineering}

The first part of the paper focuses on the study of D-brane setups that reproduce the $3 \mathrm{~d}$ dualities with linear and quadratic monopole superpotentials for SQCD with unitary and symplectic gauge groups.

Our construction is based on [8]: we consider a brane setup that engineers a $4 \mathrm{~d}$ theory, with a compact spacelike direction. Typically there are D4-, D6- and NS-branes in such setups. In addition $\mathrm{O} 4$ and $\mathrm{O} 6$ planes are added, in order to extend the analysis to the cases with real gauge groups and/or tensorial matter. We perform $T$-duality along the compact direction and study the effective $3 \mathrm{~d}$ models in the $T$-dual configuration. The $3 \mathrm{~d}$ dualities follow from the transition through infinite coupling obtained after an opportune move among the NS-branes [12]. Such moves modify the number of D3-branes that engineer the gauge sectors of the effective $3 \mathrm{~d}$ models. A common configuration corresponds to having stacks of D3-branes separated along the compact direction. This separation is associated with the presence of D1-branes that engineer the presence of interactions involving monopole operators. The simplest cases correspond, at the algebraic level, to affine Dynkin diagrams, and the affine root is associated with a linear monopole superpotential, usually referred to as the KaluzaKlein monopole superpotential. The construction has been shown in [10] to also reproduce the linear monopole superpotentials introduced in [16] for $U(1)$ models and then extended in [21] to the $U\left(N_{c}\right)$ case.

Here we introduce in this description a new ingredient, in order to also reproduce the dualities with quadratic monopole superpotentials discussed in [37]. It consists in considering compactifications with a twist by an outer automorphism of the gauge algebra. Eventually we observe that $\mathrm{D}$-branes provide a classification principle for the $3 \mathrm{~d}$ $\mathcal{N}=2$ dualities with monopole superpotentials.

The general setup is introduced in Sec. II, where we discuss general aspects of the affine and the twisted affine algebras. In Sec. III we discuss the dualities with real gauge groups. We observe that by considering the affine and the twisted affine compactifications we can reproduce the various dualities obtained in $[1,37,38]$ for $U S p\left(2 N_{c}\right)$ gauge theories involving monopole superpotentials. In Sec. IV we consider the case of $U\left(N_{c}\right)$ gauge groups. In this case we reproduce all the known dualities studied in [21,37]. Furthermore, we obtain a model that has previously been overlooked in the literature. This corresponds to SQCD with a linear (quadratic) monopole plus a quadratic (linear) antimonopole superpotential. As a check we provide the matching of the partition function along the two sides of this duality.

\section{Dualities with tensorial matter}

In the second part of the paper, corresponding to Sec. V, we study new $3 \mathrm{~d} \mathcal{N}=2$ dualities for $U\left(N_{c}\right)$ SQCD with one adjoint and $U S p\left(2 N_{c}\right)$ SQCD with one antisymmetric traceless matter field and quadratic monopole superpotential. In these cases the tensorial matter fields have a power law superpotential, which truncates the chiral ring. Moreover, we show that the quadratic monopole superpotentials are necessarily dressed by powers of the tensorial matter fields. We construct the new dualities by modifying the parent dualities obtained in $[37,39]$ for the unitary case and in [6] for the symplectic one. The deformation corresponds to a quadratic monopole superpotential in the electric and in the magnetic phase. This deformation constrains the real masses and the $R$-charges. By studying the effect of this constraint on the equality relating the partition functions of the parent theories, we arrive at a new IR identity. This new identity corresponds to the matching of the partition functions between the models with a quadratic monopole superpotential, which provides a consistency check of the new duality.

\section{THE SETUP}

\section{A. Twisted compactification and KK monopoles}

Let us consider the reduction of $4 \mathrm{~d}$ SYM with gauge group $G$ (whose Lie algebra is $g$ ) on a circle with radius $r$. If the boundary condition of the gauge field $A_{\mu}$ around the circle (say, in the direction $x^{4}$ ) is trivial, namely

$$
A_{\mu}\left(x^{0}, x^{1}, x^{2}, x^{4}+2 \pi r\right)=A_{\mu}\left(x^{0}, x^{1}, x^{2}, x^{4}\right),
$$

then the expansion of the gauge field into Fourier modes forms the untwisted affine Lie algebra $g^{(1)}$ [15]. More generally, one may consider the boundary condition (see [40] for an extensive discussion)

$$
A_{\mu}\left(x^{0}, x^{1}, x^{2}, x^{4}+2 \pi r\right)=\sigma\left(A_{\mu}\left(x^{0}, x^{1}, x^{2}, x^{4}\right)\right)
$$

where $\sigma$ is an outer automorphism of the Lie algebra $g$. For the Lie algebras $g=A_{N}, D_{N}, E_{6}$, the element $\sigma$ can be of order $L=2$, and for $g=D_{4}, \sigma$ can be of order $L=3$. The Lie algebra $g$ can be decomposed into the direct sum of the eigenspaces $\mathcal{G}_{n}$ (with $n=0, \ldots, L-1$ ) associated with the eigenvalues $\mathrm{e}^{2 \pi \mathrm{in} / L}$ of $\boldsymbol{\sigma}$ :

$$
\boldsymbol{\sigma}(h)=\mathrm{e}^{2 \pi \mathrm{i} n / L} h, \quad \text { for } h \in \mathcal{G}_{n} .
$$

The mode expansion of $A_{\mu}$ can be written as [40]

$$
\begin{aligned}
A_{\mu}^{i}\left(x^{4}\right) T^{i} & =\sum_{m \in \mathbb{Z}} \sum_{n=0}^{L-1} A_{\mu}^{i,(m, n)} \exp \left(-\mathrm{i} \frac{x^{4}}{r}\left(m+\frac{n}{L}\right)\right) T^{i} \\
& =\sum_{m \in \mathbb{Z}} \sum_{n=0}^{L-1} A_{\mu}^{i,(m, n)} T_{m+\frac{n}{L}}^{i}
\end{aligned}
$$

where $T_{i}$ (with $i=1, \ldots, \operatorname{dim} g$ ) are the Lie algebra generators, and we define 


$$
T_{m+\frac{n}{L}}^{i}:=\exp \left(-\mathrm{i} \frac{x^{4}}{r}\left(m+\frac{n}{L}\right)\right) T^{i}
$$

If $\boldsymbol{\sigma}$ is trivial, then $T_{m}^{a}$ form a set of the generators of the untwisted affine Lie algebra $g^{(1)}$; we refer to this case as an untwisted compactification. However, if $\boldsymbol{\sigma}$ is nontrivial and is of order $L=2,3$, then $T_{m+\frac{n}{L}}^{i}$ form a set of the generators of the twisted affine Lie algebra $g^{(L)}$; we refer to this case as a twisted compactification. In the following discussion, we focus only on the case of $L=2$, with the twisted affine Lie algebras $A_{2 N-1}^{(2)}, A_{2 N}^{(2)}$, and $D_{N+1}^{(2)}$.

In the three-dimensional limit where $r \rightarrow 0$, the gauge algebra $g$ reduces to a smaller Lie algebra $\mathcal{G}_{0}$ since in this case we do not have a Kaluza-Klein mass term. The rank $r^{\prime}$ of $\mathcal{G}_{0}$ can be smaller than that of $G$. The simple roots $\beta_{a}$ (with $a=1, \ldots, r^{\prime}$ ) of $\mathcal{G}_{0}$, together with the lowest negative weight $\beta_{0}$ of $\mathcal{G}_{1}$, form the Dynkin diagram of the twisted affine Lie algebra $g^{(L)}$.

An instanton on $\mathbb{R}^{3} \times S^{1}$ can be regarded as a composite that contains fundamental monopoles as constituents [4147]. Each of the fundamental monopoles consists of four zero modes-namely, three associated with its position and one associated with the phase - and is labeled by the coroot $\beta_{a}^{*}$ (with $a=0, \ldots, r^{\prime}$ ). Any other monopole configuration is a composite of such fundamental monopoles. The aforementioned instanton configuration is characterized by a set of non-negative integers $n_{a}$ (with $a=0, \ldots, r^{\prime}$ ) which count the magnetic charge of each fundamental monopole $\beta_{a}^{*}$. Such an instanton can contribute nontrivially to the effective potential of the theory. For example, for the $\mathcal{N}=1^{*}$ supersymmetric theory on $\mathbb{R}^{3} \times S^{1}$ with a twisted boundary condition, the instanton contribution to the holomorphic superpotential is given by [40]

$$
W=\frac{2}{\beta_{0}^{2}} \eta \mathrm{e}^{\frac{4 \pi i \tau}{L \beta_{0}^{2}}+\beta_{0}^{*} \cdot X}+\sum_{a=1}^{r^{\prime}} \frac{2}{\beta_{a}^{2}} \mathrm{e}^{\beta_{a}^{*} \cdot X}
$$

where $X$ is the adjoint chiral field in the theory and $\tau$ is the holomorphic coupling of the theory.

\section{B. Brane configurations}

From the string theory perspective, the instanton configuration discussed above can be realized from the brane system containing D0- and D4-branes, possibly with the presence of the orientifold four-plane, where four-branes span $\mathbb{R}^{3} \times S^{1}$. Upon using $T$ duality along the $S^{1}$ direction, we obtain the system consisting of D1-branes stretching between D3-branes, possibly with the presence of orientifold three-planes. The $T$-dual radius is $R=\frac{\alpha^{\prime}}{r}$. The effect of $T$-duality on various types of orientifold four-planes is

\begin{tabular}{|c|c|}
\hline Orientifold & $T$-duality \\
\hline$\widetilde{\mathrm{O}}^{-}$ & $\mathrm{O}^{-} \& \widetilde{\mathrm{O}_{3}}$ \\
\hline $\mathrm{O}^{+}$ & $\mathrm{O}^{+} \& \mathrm{O}^{+}$ \\
\hline $\mathrm{O}^{-}$ & $\mathrm{O}^{-} \& \mathrm{O}^{-}$ \\
\hline$\widetilde{\mathrm{O}}^{+}$ & $\widetilde{\mathrm{O}}^{+} \& \mathrm{O}^{+}$ \\
\hline
\end{tabular}
tabulated below.

The brane configurations that give rise to the untwisted affine Dynkin diagrams as quiver gauge theories on the D1branes are tabulated below [47] (see also [48]). The affine node is denoted in black.

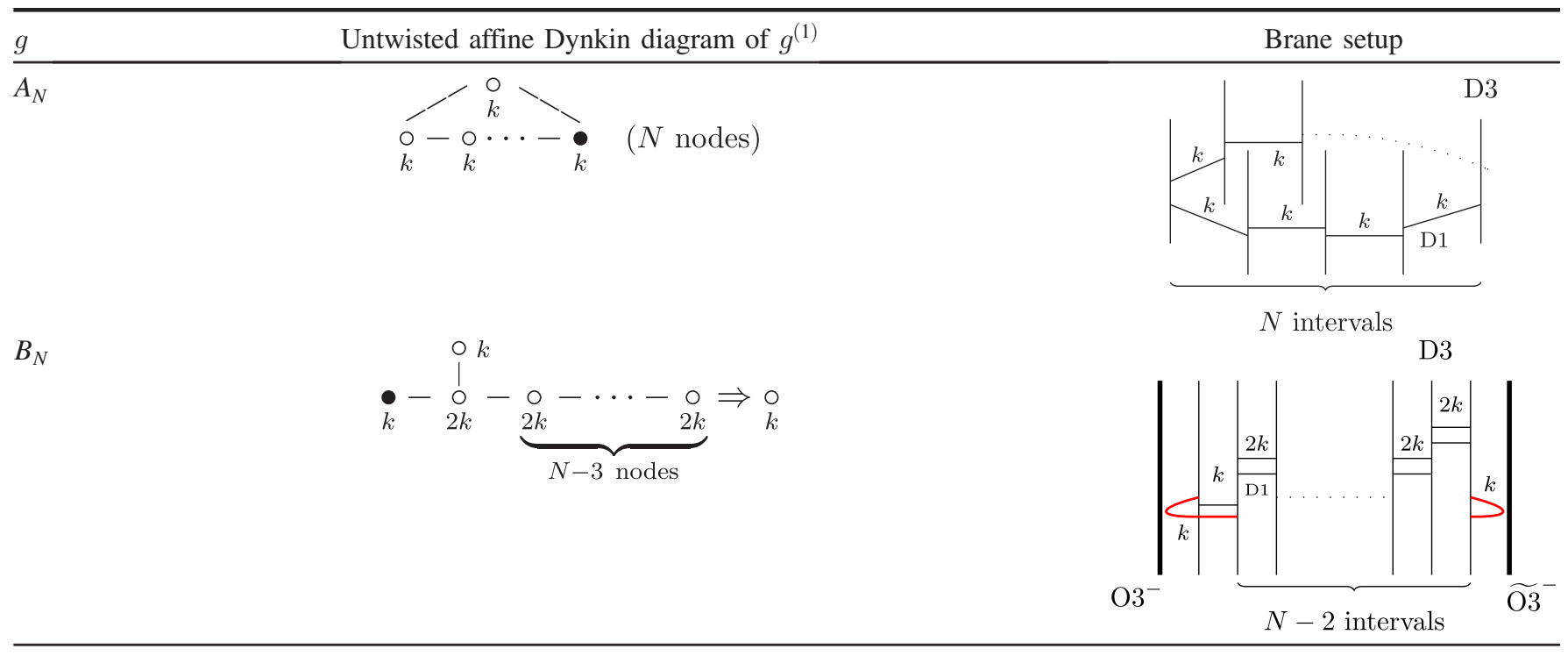

(Table continued) 


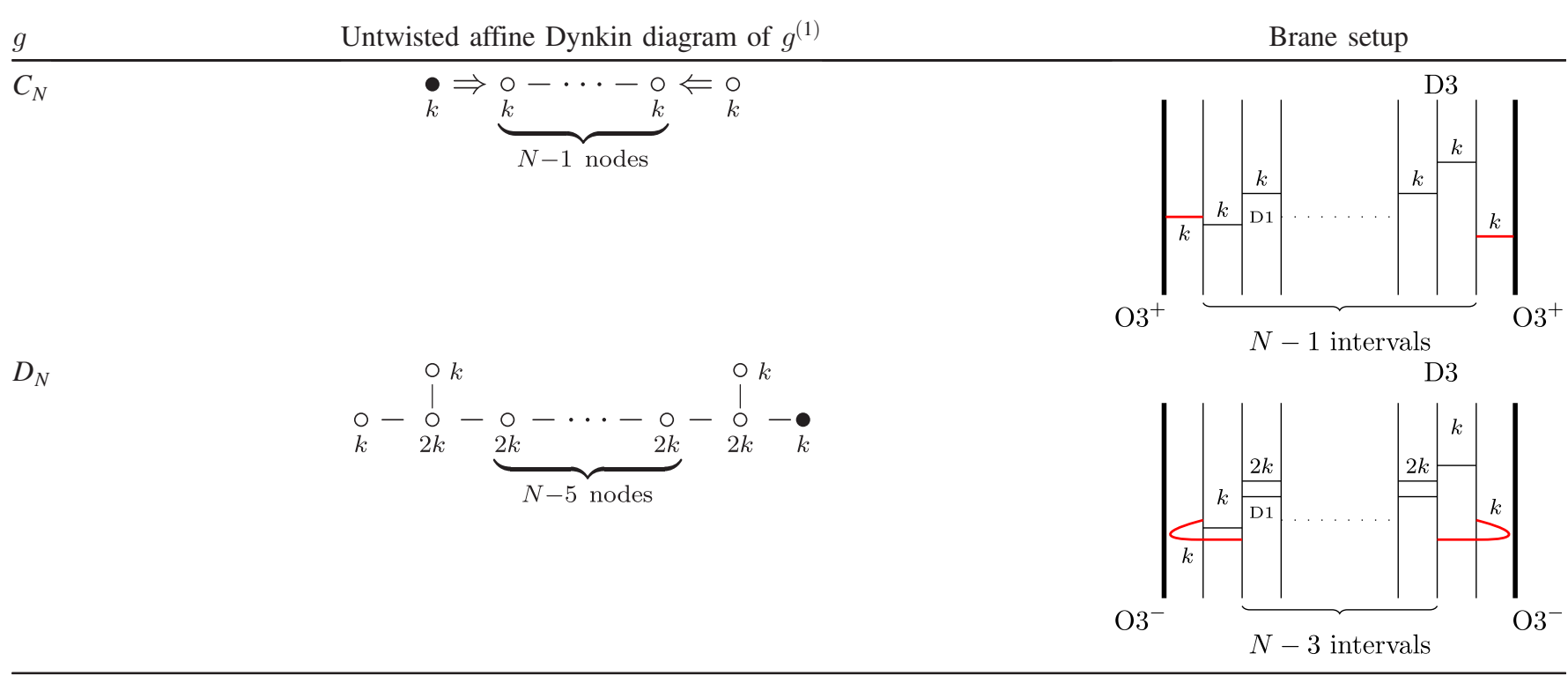

It is worth noting the relation between the "boundary" of the Dynkin diagram and the type of the orientifold planes [47]. In particular,

(i) the bifurcation corresponds to $\mathrm{O}^{-}$;

(ii) the double arrow going into the main body of the quiver corresponds to $\mathrm{O}^{+}$; and

(iii) the double arrow going out of the main body of the quiver corresponds to $\mathrm{O}^{-}$.

For the twisted case, we only focus on the twisted affine Lie algebras $A_{2 N-1}^{(2)}, A_{2 N}^{(2)}$, and $D_{N+1}^{(2)}$. Their Dynkin diagrams can be realized on the world volume of the D1-branes in the following brane setup [47]. Observe that the types of orientifold three-planes are in accordance with the rules stated above.

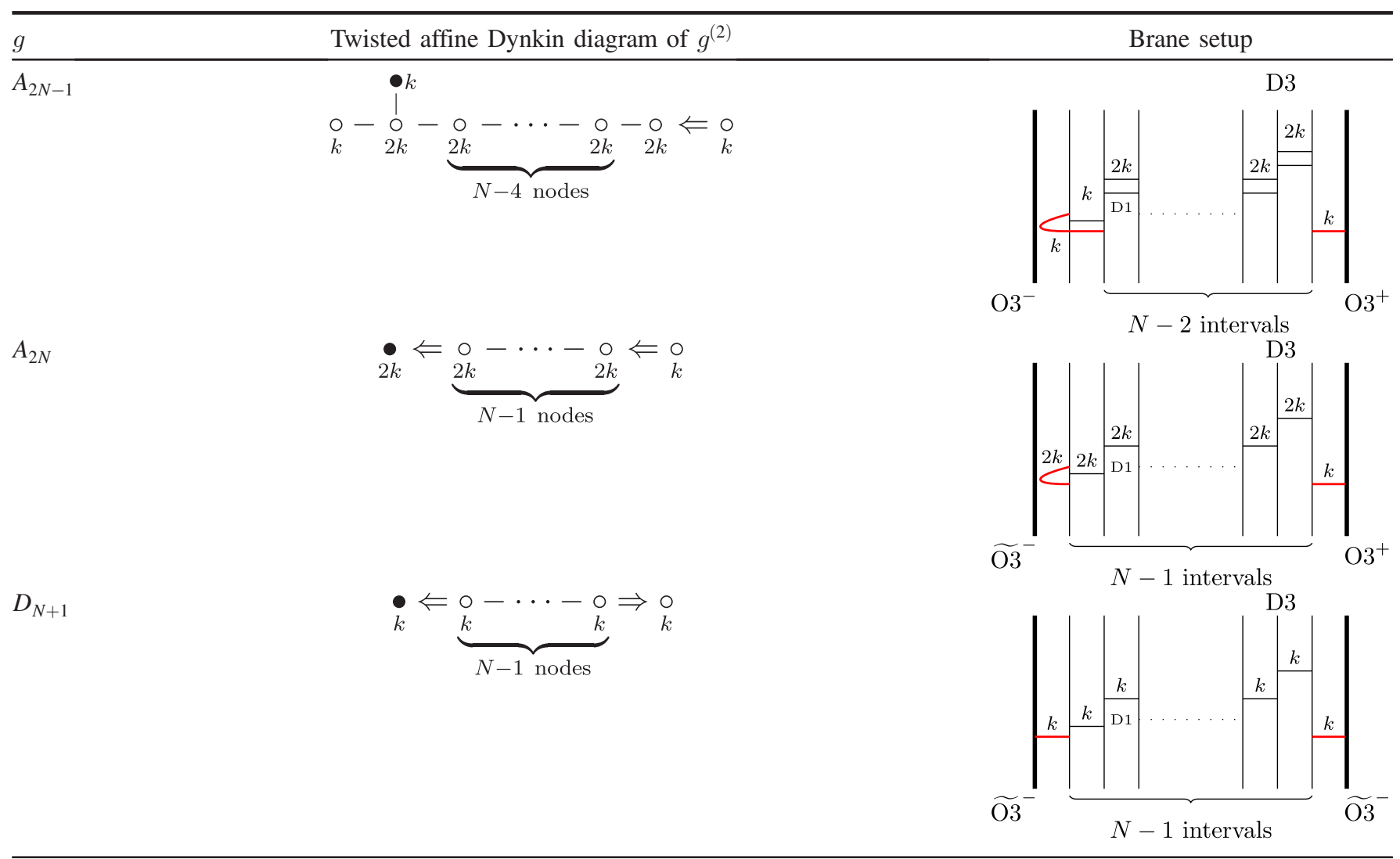


As pointed out in [47], in the twisted affine cases, we need to turn on the Wilson line in the compact direction of the world volume of the D4-branes in order to enhance the algebra $\mathcal{G}_{0}$ to the full twisted Lie algebra $g^{(L)}$.

\section{DUALITIES FOR SYMPLECTIC GAUGE GROUPS}

In this section we discuss the $3 \mathrm{~d}$ dualities for $U S p\left(2 N_{c}\right)$ SQCD obtained by compactifications of $4 \mathrm{~d}$ theories on $S^{1}$ in the presence of orientifolds. We study both the affine and the twisted affine configurations. We recover the various models discussed in the literature, namely, Aharony duality and dualities with linear and quadratic monopole superpotentials.
In order to fix the notations and the geometric setup, we consider in $4 \mathrm{~d}$ an NS-brane extended along $x_{0,1,2,3,4,5}$ and an NS'-brane extended along $x_{0,1,2,3,8,9}$. The two fivebranes are separated along $x_{6}$. They are connected along this directions by a stack of D4-branes. These last ones are finite along $x_{6}$, and they fill $x_{0,1,2,3}$. The flavor is obtained by adding to the picture a stack of D6-branes extended along $x_{0,1,2,3,7,8,9} \cdot T$-duality is performed along $x_{3}$. In this way the NS-branes are compact along $x_{3}$, while the D4- and D6-branes become D3 and D5, respectively; they are not extended anymore along $x_{3}$. To this picture we can add a pair of orientifolds, as discussed above.

Our brane description distinguishes three possible $3 \mathrm{~d}$ $\mathcal{N}=2$ gauge theories with symplectic gauge group and fundamental matter. They are summarized in the table below. Let us study these three cases separately.

\begin{tabular}{lccc}
\hline$G_{\text {ele }}$ & $G_{\mathrm{mag}}$ & $W_{\mathrm{ele}}$ & $W_{\mathrm{mag}}$ \\
\hline$U S p\left(2 N_{c}\right)$ & $W=0$ & $W=M_{q q}+y Y$ \\
$U S p\left(2 N_{c}\right)$ & $W=Y$ & $W=M_{q q}+y$ \\
$U S p\left(2 N_{c}\right)$ & $W=Y^{2}$ & $W=M_{q q}+y^{2}$
\end{tabular}

\section{A. $U \operatorname{Sp}\left(2 N_{c}\right)$ with $W=0$}

This case corresponds to the original Aharony duality discussed in [38]. In the brane picture it corresponds to the setup with an $\mathrm{O}^{+}$plane at $x_{3}=0$ and an $\mathrm{O}^{-}$at $x_{3}=\pi R$. Such an orientifold boundary condition corresponds to the twisted affine algebra $A_{2 N_{c}-1}^{(2)}$ :

$$
0-\stackrel{i}{i}-\underbrace{0-0-\cdots-0-0}_{\left(N_{c}-3\right) \text { nodes }} \Leftarrow 0 \text {. }
$$

Observe that there is also a configuration with the two orientifolds exchanged. Such a configuration corresponds to turning on opportune Wilson lines in the $4 \mathrm{~d}$ setup.

In this brane setup we consider $N_{c}$ D3 and $N_{f}$ D5 on top of $\mathrm{O}^{+}$at $x_{3}=0$, while we do not have any further brane at $x_{3}=\pi R$. This gives rise to the $U S p\left(2 N_{c}\right)$ gauge theory with $2 N_{f}$ fundamentals.

Let us now discuss the brane configuration after the transition through infinite coupling. At $x_{3}=0$, where $\mathrm{O}^{+}$ is located, we have $N_{f}-N_{c}-1$ physical D3-branes, where -1 is there to cancel the charge of the $\mathrm{O}^{+}$plane. On the other hand, at $x_{3}=\pi R$, where $\mathrm{O}^{-}$is located, we have one physical D3-brane to cancel the charge of $\mathrm{O}^{-}$. This configuration gives rise to a $\operatorname{USp}\left(2\left(N_{f}-N_{c}-1\right)\right)$ gauge theory with $2 N_{f}$ chirals at $x_{3}=0$, and a pure $S O(2)$ gauge sector at $x_{3}=\pi R$. There is also an interaction ${ }^{1}$

\footnotetext{
${ }^{1}$ A monopole $Y$ is identified here with a Coulomb branch coordinate $\Sigma$. On the Coulomb branch the latter are holomorphic combinations $\Sigma \equiv i \varphi+\frac{\sigma}{g^{2}}$ of the dual photon $\varphi$ and of the real scalar $\sigma$ in the vector multiplet, where $g$ represents the gauge coupling.
} 


$$
W=\mathrm{e}^{\Sigma-\Sigma_{1}}
$$

between the monopole $y=\mathrm{e}^{-\Sigma_{1}}$ of the dual $\operatorname{USp}\left(2\left(N_{f}-\right.\right.$ $\left.N_{c}-1\right)$ ) gauge group and the monopole $Y=\mathrm{e}^{\Sigma}$ of the $\mathrm{SO}(2)$ sector.

Observe that in this case, in the absence of D-branes in the electric sector we can consider the large $T$-dual radius limit, describing a pure $3 \mathrm{~d}$ gauge theory. On the magnetic side we can dualize the $S O(2)$ gauge sector, and indeed we keep the singlet $Y$, acting with the superpotential (3.2), corresponding to the interaction $W=y Y$ of the Aharony duality.

\section{B. $U S p\left(2 N_{c}\right)$ with $W=Y$}

This case corresponds to the affine $C_{N_{c}}^{(1)}$ case, corresponding to the circle compactification of the $4 \mathrm{~d}$ $\operatorname{USp}\left(2 N_{c}\right)$ theory and of its Intriligator-Pouliot dual description, whose affine Dynkin diagram is

$$
\circ \Rightarrow \underbrace{0-\cdots-0}_{N_{c}-1 \text { nodes }} \Leftarrow \circ
$$

In terms of branes, we have an $\mathrm{O}^{+}$plane at $x_{3}=0$ and an $\mathrm{O}^{+}$at $x_{3}=\pi R$. In this brane setup we consider $N_{c} \mathrm{D} 3$ and $N_{f}-1$ D5 at $x_{3}=0$ (with their images), while we have one D5 $x_{3}=\pi R$ (with its image). This system gives rise to the $\operatorname{USp}\left(2 N_{c}\right)$ gauge theory with $2 N_{f}$ fundamentals with $W=Y$, where the monopole superpotential can be read from the brane configuration at $x_{3}=\pi R$.

Let us now discuss the brane configuration after the transition through infinite coupling. At $x_{3}=0$, where one of the $\mathrm{O}^{+}$planes is located, we have $\left(N_{f}-1\right)-N_{c}-1$ physical D3-branes, where the last -1 is there to cancel the charge of $\mathrm{O}^{+}$. This gives rise to a $U S p\left(2\left(N_{f}-N_{c}-2\right)\right)$ gauge theory with $2 N_{f}$ fundamentals. At $x_{3}=\pi R$, where the other $\mathrm{O}^{+}$is located, we have $(1-0)-1=0$ D3branes, where 1 and 0 denote numbers of D5- and D3branes before the transition and the last -1 is there to cancel the charge of $\mathrm{O}^{+}$. The absence of D3-branes at $x_{3}=\pi R$ for both the electric and the magnetic descriptions allows us to place the extra D5-branes from this position to $x_{3}=0$. This system gives rise to the dual theory, which is the $\operatorname{USp}\left(2\left(N_{f}-N_{c}-2\right)\right)$ gauge theory with $2 N_{f}$ fundamentals, singlets $M$ and $W=M q q+y$, where the monopole superpotential can be read from the brane configuration at $x_{3}=\pi R$.

\section{C. $U S p\left(2 N_{c}\right)$ with $W=Y^{2}$}

This case corresponds to another twisted affine compactification. At the geometric level we have an $\mathrm{O}^{+}$plane at $x_{3}=0$ and an $\widetilde{\mathrm{O}}^{-}$at $x_{3}=\pi R$. Such an orientifold boundary condition corresponds to the twisted affine algebra $A_{2 N_{c}}^{(2)}$ :

$$
\circ \Leftarrow \underbrace{\circ-\circ-\cdots-\circ-0}_{\left(N_{c}-1\right) \text { nodes }} \Leftarrow 0 .
$$

Observe that there is also a configuration with the two orientifolds exchanged. Such a configuration corresponds to turning on opportune Wilson lines in the $4 \mathrm{~d}$ setup.

In this brane setup we consider $N_{c}$ D3 and $N_{f}$ D5 (with their images), along with $\mathrm{O}^{+}$, at $x_{3}=0$, while we have a half-physical D3-brane stuck on the $\widetilde{\mathrm{O}}^{-}$plane at $x_{3}=\pi R$. This gives rise to the $\operatorname{USp}\left(2 N_{c}\right)$ gauge theory with $2 N_{f}$ fundamentals and $W=Y^{2}$, where the monopole superpotential can be read off from the configuration at $x_{3}=\pi R$.

After the transition through infinite coupling we are left with $N_{f}-N_{c}-1 \mathrm{D} 3$-branes at $x_{3}=0$, where -1 is there to cancel the charge of $\mathrm{O3}^{+}$. There is a half-physical D3brane stuck on $\widetilde{\mathrm{O}}^{-}$at $x_{3}=\pi R$. This system gives rise to the dual theory, namely, the $\operatorname{USp}\left(2\left(N_{f}-N_{c}-1\right)\right)$ gauge theory with $2 N_{f}$ fundamentals, singlets $M$ and the superpotential $W=M q q+y^{2}$.

As a final remark, it is worth mentioning that the duality discussed in this section can be obtained from that discussed in Sec. III A as follows. One may deform the electric theory in Sec. III A by adding the term $Y^{2}$ to the superpotential. Upon using the duality discussed in Sec. III A, we obtain the superpotential $M q q+y Y+Y^{2}$ in the magnetic theory, where $Y$ is now an elementary field that can be integrated out, which leads to the superpotential $M q q-\frac{1}{4} y^{2}$. The factor $-1 / 4$ can be easily absorbed into $y$ by a field redefinition of $y$. We therefore obtain the superpotential discussed in the preceding paragraph. This argument has in fact also been used in Sec. 5.2 of [37].

\section{DUALITIES FOR UNITARY GAUGE GROUPS}

In this section we study unitary gauge groups, corresponding to placing $N_{c}$ D3-branes and $N_{f}$ D5-branes at $x_{3}=\frac{\pi}{2} R$. Depending on the choice of the orientifolds we also place other D3- or D5-branes at $x_{3}=0$ and $x_{3}=\pi R$. By exchanging the position of the NS-branes we generate the dual description. We can summarize the results of this section in the following table. 


\begin{tabular}{lccc}
\hline$G_{\text {ele }}$ & $G_{\mathrm{mag}}$ & $W_{\mathrm{ele}}$ & $W=M q \tilde{q}+T_{+} t_{+}+T_{-} t_{-}$ \\
\hline$U\left(N_{c}\right)$ & $U\left(N_{f}-N_{c}\right)$ & $W=0$ & $W=M q \tilde{q}+t_{+}+t_{-}$ \\
$U\left(N_{c}\right)$ & $U\left(N_{f}-N_{c}-2\right)$ & $W=T_{+}+T_{-}$ & $W=M q \tilde{q}+t_{-}+T_{-} t_{+}$ \\
$U\left(N_{c}\right)$ & $U\left(N_{f}-N_{c}-1\right)$ & $W=T_{+}^{2}+T_{-}^{2}$ \\
$U\left(N_{c}\right)$ & $W=T_{+}^{2}$ & $W=M q \tilde{q}+t_{+}^{2}+t_{-}^{2}$ \\
$U\left(N_{c}\right)$ & $W\left(N_{f}-N_{c}\right)$ & $W T_{+}^{2}+T_{-}$ \\
& $U\left(N_{f}-N_{c}-1\right)$ & $W$
\end{tabular}

As already anticipated in the Introduction, most of the models have already been discussed in the literature. However, there is a new case, so far overlooked, corresponding to $U\left(N_{c}\right)$ SQCD with $W=T_{+}^{2}+T_{-}$. Observe that a full classification should have nine inequivalent cases. The other three cases that we did not discuss here correspond to the pairs $\left(\mathrm{O3}^{-}, \widetilde{\mathrm{O}}^{-}\right),\left(\mathrm{O}^{-}, \mathrm{O}^{+}\right)$and $\left(\mathrm{O3}^{+}, \widetilde{\mathrm{O}}^{-}\right)$. These cases can be obtained by charge conjugation on $\left(\widetilde{\mathrm{O}^{-}}{ }^{-}, \mathrm{O}^{-}\right)$, $\left(\mathrm{O}^{+}, \mathrm{O}^{-}\right)$and $\left(\widetilde{\mathrm{O}}^{+}, \mathrm{O}^{+}\right)$, respectively.

In the following we discuss the various cases separately, showing how to construct the $3 \mathrm{~d}$ dualities from the brane picture in each case.

\section{A. $U\left(N_{c}\right)$ with $W=0$ : Aharony duality}

Aharony duality can be constructed by reducing a $4 \mathrm{~d}$ $S O(2 N)$ gauge theory with $N_{f}$ flavors on $S^{1}$ and considering the vacuum corresponding to $N_{c}$ D3 and $N_{f}$ D5 at $x_{3}=\frac{\pi}{2} R$. The corresponding $4 \mathrm{~d}$ theory on $S^{1}$ has a monopole superpotential $W=\eta Z$, where $Z$ is the KK monopole operator $^{2}[3]$.

Since we do not have any extra D3 or D5 at $x_{3}=0, \pi R$, it signals the fact that we can send the radius and the monopole superpotential to zero. This is in agreement with the discussion below (5.2) of [3]. The resulting theory is thus a $3 \mathrm{~d} U\left(N_{c}\right)$ gauge theory with $N_{f}$ flavors and zero superpotential.

\footnotetext{
${ }^{2}$ Semiclassically, this corresponds to $Z \sim \mathrm{e}^{\Sigma_{1}+\Sigma_{2}}$ in the notation of [3].
}

In the dual picture we have $N_{f}-N_{c}$ D3 and $N_{f}$ D5 at $x_{3}=\frac{\pi}{2} R$. Moreover, we have a D3 and its image at $x_{3}=0$ and at $x_{3}=\pi R$. In this case we have to dualize these $S O(2)=U(1)$ gauge theories. In the dual picture the monopole corresponds to a singlet, and it can be identified with the electric monopole acting as a singlet in this dual phase. This is compatible with the claim that this brane picture represents the dual phase of Aharony duality.

\section{B. $U\left(N_{c}\right)$ with $W=T_{+}+T_{-}$}

This duality has already been studied in [21], and it corresponds to the reduction of a $4 \mathrm{~d} U \operatorname{Sp}\left(2 N_{c}\right) \mathrm{SQCD}$ with $2 N_{f}$ fundamentals. Upon putting this theory on $S^{1}$, a superpotential $W=\eta Y$ is generated. In the electric theory one needs to consider a vacuum with $N_{c}$ D3 and $N_{f}-2$ D5 at $x_{3}=\frac{\pi}{2} R$. Moreover, there is a pair of one D5-brane and its image at both $x_{3}=0$ and $x_{3}=\pi R$.

The dual picture has $N_{f}-N_{c}-2$ D3 and $N_{f}-2$ D5 at $x_{3}=\frac{\pi}{2} R$. and again a pair of one D5-brane and its image at both $x_{3}=0$ and $x_{3}=\pi R$. The absence of D3-branes at $x_{3}=0$ and $x_{3}=\pi R$ in both phases allows us to recollect all the D5 at $x_{3}=\frac{\pi}{2} R$ in both phases. Furthermore, the monopole superpotential can be read from the spectrum of D1-branes connecting the stack of D3-branes at the orientifolds.

\section{C. $U\left(N_{c}\right)$ with $W=T_{+}$}

We start by reducing the $4 \mathrm{~d} U S p\left(2 N_{c}\right)$ gauge theory with $2 N_{f}$ fundamentals and its dual on a circle. The brane 
system consists of an $\mathrm{O}^{+}$plane at $x_{3}=0$ and an $\mathrm{O}^{-}$ plane at $x_{3}=\pi R$. The electric theory on $S^{1}$ has a superpotential $W=\eta Y$, and the dual theory has gauge group $U S p\left(2 N_{f}-2 N_{c}-4\right)$. Such an orientifold boundary condition corresponds to the twisted affine algebra $A_{2 N_{c}-1}^{(2)}$ :

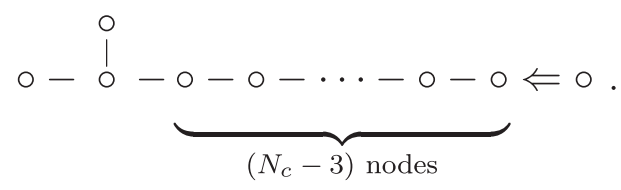

For our aims the configuration with the two orientifolds exchanged is completely equivalent, and it corresponds to turning on opportune Wilson lines in the $4 \mathrm{~d}$ setup.

In the electric theory we have $N_{c}$ D3 and $N_{f}-1$ D5 at $x_{3}=\frac{\pi}{2} R$. We also consider one D5-brane and its image at $x_{3}=0$, while we do not have any D-brane at $x_{3}=\pi R$.

In the dual model we have $N_{f}-N_{c}-1 \mathrm{D} 3$ and $N_{f}-1$ D5 at $x_{3}=\frac{\pi}{2} R$, along with one D5 and its image at $x_{3}=0$ and one D3 and its image at $x_{3}=\pi R$. We are free to connect all the D5 at $x_{3}=\frac{\pi}{2} R$ in both phases and to dualize the $S O(2)$ gauge theory into a scalar. The final duality relates a $U\left(N_{c}\right)$ gauge theory with $N_{f}$ pairs of fundamentals and antifundamentals with monopole superpotential $W=T_{+}$with a $U\left(N_{f}-N_{c}-1\right)$ gauge theory with $N_{f}$ pairs of dual fundamentals and antifundamentals, with superpotential $W=M q \tilde{q}+t_{-}+t_{+} T_{-}$where $M$ corresponds to the meson of the electric theory and $T_{-}$is the dual photon of the $S O(2)$ gauge theory and has the same quantum numbers of the antimonopole of the electric theory.

Let us end this subsection by mentioning a puzzle regarding the twisted compactification in this case. As we mentioned at the beginning, we start from the $U S p\left(2 N_{c}\right)$ gauge theory on $S^{1}$. There are two options to obtain such a gauge algebra from $4 \mathrm{~d}$, namely,

(1) $A_{2 \ell}=s u(2 \ell+1) \rightarrow C_{\ell}=u s p(2 \ell)$; or

(2) $D_{\ell+1}=s o(2 \ell+2) \rightarrow C_{\ell}=u s p(2 \ell)$.

For option 1, the $4 \mathrm{~d}$ Seiberg duality between an $S U\left(2 N_{c}+1\right)$ gauge theory with $2 N_{f}$ flavors and an $S U\left(2 N_{f}-2 N_{c}-1\right)$ gauge theory with $2 N_{f}$ flavors becomes a duality between a $U S p\left(2 N_{c}\right)$ gauge theory and a $U S p\left(2 N_{f}-2 N_{c}-2\right)$ gauge theory; however, the latter is not $U S p\left(2 N_{f}-2 N_{c}-4\right)$ as expected. For option 2 , the $4 \mathrm{~d}$ Seiberg duality between an $S U\left(2 N_{c}+2\right)$ gauge theory with $2 N_{f}$ flavors and an $S U\left(2 N_{f}-2 N_{c}-2+4\right)=$ $S U\left(2 N_{f}-2 N_{c}+2\right)$ gauge theory with $2 N_{f}$ flavors becomes a duality between a $U S p\left(2 N_{c}\right)$ gauge theory and a $U S p\left(2 N_{f}-2 N_{c}\right)$ gauge theory; however, the latter is not $U S p\left(2 N_{f}-2 N_{c}-4\right)$ as expected. One possibility to resolve this puzzle is that in this brane setup there is a Wilson line that could break the $\operatorname{USp}\left(2 N_{f}-2 N_{c}-2\right)$ gauge group to the $U S p\left(2 N_{f}-2 N_{c}-4\right)$ gauge group [or from the $U S p\left(2 N_{f}-2 N_{c}\right)$ gauge group to the $U S p\left(2 N_{f}-2 N_{c}-4\right)$ gauge group]. We leave this for future work.

\section{D. $U\left(N_{c}\right)$ with $W=T_{+}^{2}+T_{-}^{2}$}

The $3 \mathrm{~d}$ duality in this case can be realized by starting from the following $4 \mathrm{~d}$ theories on $S^{1}$ with a special orthogonal gauge algebra. The latter can be obtained from $4 \mathrm{~d}$ Seiberg duality by twisted compactification as follows. Let us use the nontrivial outer-automorphism of the $A_{2 \ell-1}=s u(2 \ell)$ algebra to twist and obtain the $B_{\ell}=$ so $(2 \ell+1)$ algebra: The Seiberg duality between the $S U(2 \ell)$ gauge theory with $2 N_{f}$

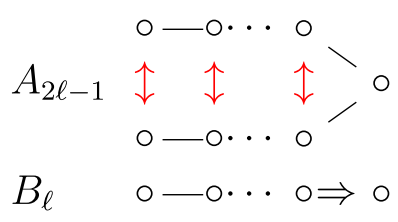

flavors and the $S U\left(2 N_{f}-2 \ell\right)$ gauge theory with $2 N_{f}$ flavors and singlets then becomes a duality between a theory with the $s o(2 \ell+1)$ gauge algebra and a theory with the $s o\left(2 N_{f}-2 \ell+1\right)$ gauge algebra after twisting. In this paper, we do not go into any further detail of the duality between theories with the orthogonal gauge algebra.

The brane system of such theories with the $B$-type orthogonal gauge algebras contains a pair of $\widetilde{\mathrm{O}}^{-}$planes, one at $x_{3}=0$ and the other at $x_{3}=\pi R$. Recall that on each $\tilde{\mathrm{O}}^{-}$plane, there is a half D3-brane stuck there. The orientifold boundary condition corresponds to the twisted affine algebra $D_{N_{c}+1}^{(2)}$ :

$$
\underbrace{\circ \Leftarrow \circ-\circ-\cdots-\circ-\circ \Rightarrow 0}_{\left(N_{c}+1\right) \text { nodes }} .
$$

In the electric theory we have $N_{c}$ D3 and $N_{f}$ D5 at $x_{3}=\frac{\pi}{2} R$ : the gauge theory corresponds to $U\left(N_{c}\right)$ with $N_{f}$ pairs of fundamentals and antifundamentals and superpotential $W=T_{+}^{2}+T_{-}^{2}$ corresponding to the spectrum of D1-branes connecting the D3-branes at the orientifold and the D3-branes on the stack.

In the dual configuration we have $N_{f}-N_{c}$ D 3 and $N_{f}$ D5 at $x_{3}=\frac{\pi}{2} R$, a single D3 at $x_{3}=0$ and another at $x_{3}=\pi R$. The gauge theory corresponds to $U\left(N_{f}-N_{c}\right)$ with $N_{f}$ pairs of fundamentals and antifundamentals and superpotential $W=M q \tilde{q}+t_{+}^{2}+t_{-}^{2}$.

It is worth noting that the duality discussed in this section can also be obtained from that in Sec. IVA by a similar deformation as discussed in Sec. III C.

\section{E. $U\left(N_{c}\right)$ with $W=T_{+}^{2}$}

This duality can be constructed by reducing a $4 \mathrm{~d}$ model with $s o\left(2 N_{c}+1\right)$ gauge algebra and $2 N_{f}$ vectors on $S^{1}$. 
The brane setup contains an ${\widetilde{\mathrm{O3}^{-}}}^{-}$at $x_{3}=0$ where there is a half D3-brane stuck there, together with an $\mathrm{O}^{-}$at $x_{3}=\pi R$. We consider the vacuum corresponding to $N_{c}$ $\mathrm{D} 3$ and $N_{f} \mathrm{D} 5$ at $x_{3}=\frac{\pi}{2} R$. This electric theory corresponds to a $U\left(N_{c}\right)$ model with $N_{f}$ pairs of fundamentals and antifundamentals and superpotential $W=T_{+}$. As discussed above we consider a decoupling limit without the generation of any monopole superpotential arising from the $\mathrm{O}^{-}$plane.

The dual model is obtained by exchanging the position of the NS-branes, and it corresponds to considering $N_{f}-N_{c}$ D3-branes and $N_{f}$ D5-branes at $x_{3}=\frac{\pi}{2} R$ and again a half D3 stuck on $\widetilde{\mathrm{O}}^{-}$at $x_{3}=0$. Furthermore, we have one D3-brane and its image at $x_{3}=\pi R$ on the $3^{-}$ plane. The total amount of D3-branes in this setup corresponds to the total amount of D4 in the 4d theory, as it should. Indeed the dual $4 \mathrm{~d}$ model corresponds to a theory with algebra $s o\left(2 N_{f}-2 N_{c}+3\right)$; this is because we can recollect all $N_{f}-N_{c}+1 \mathrm{D} 3$-branes on the $\widetilde{\mathrm{O}}^{-}$plane. The $S O(2)$ gauge theory at $x_{3}=\pi R$ can be dualized to a scalar, and this scalar corresponds to the electric monopole acting as a singlet in the dual phase. All in all, the dual model corresponds to a $U\left(N_{f}-N_{c}\right)$ gauge theory with $N_{f}$ pairs of fundamentals and antifundamentals and superpotential $W=t_{-}^{2}+T_{-} t_{+}$, with $T_{-}$the singlet obtained by dualizing the $S O(2)$ gauge theory.

\section{F. $U\left(N_{c}\right)$ with $W=T_{+}^{2}+T_{-}$}

The $3 \mathrm{~d}$ duality in this case can be realized by starting from the following $4 \mathrm{~d}$ theories on $S^{1}$ with a symplectic gauge algebra and a quadratic monopole superpotential:

(A) $\operatorname{USp}\left(2 N_{c}\right) \operatorname{SQCD}$ with $2 N_{f}$ chirals and $W=Y^{2}$.

(B): $U S p\left(2 N_{f}-2 N_{c}-2\right) \operatorname{SQCD}$ with $2 N_{f}$ chirals,

$$
\text { singlets } M \text { and } W=M q q+\hat{Y}^{2} \text {. }
$$

We may obtain such a duality from $4 \mathrm{~d}$ Seiberg duality by twisted compactification as follows. Let us use the nontrivial outer-automorphism of the $A_{2 \ell}=s u(2 \ell+1)$ algebra to twist and obtain the $C_{\ell}=u s p(2 \ell)$ algebra. The Seiberg duality between the $S U\left(2 N_{c}+1\right)$ gauge theory with $2 N_{f}$ flavors and the $S U\left(2 N_{f}-2 N_{c}-1\right)$ gauge theory with $2 N_{f}$ flavors and singlets then becomes a duality between the $U S p\left(2 N_{c}\right)$ gauge theory and the $U S p\left(2 N_{f}-2 N_{c}-2\right)$ gauge theory after twisting. These are indeed the gauge groups in (4.3), as required.

The brane system contains an $\mathrm{O}^{+}$at $x_{3}=0$ (or at $x_{3}=\pi R$ ) and an $\widetilde{\mathrm{O}}^{-}$at $x_{3}=\pi R$ (or at $x_{3}=0$ ). In the presence of D3-branes this gives rise to the superpotential $W=T_{+}^{2}+T_{-}$(or $W=T_{-}^{2}+T_{+}$). This case is interesting because it has been overlooked so far in the literature, while it seems a natural possibility to investigate it in the brane setup. The orientifold boundary condition corresponds to the twisted affine algebra $A_{2 N_{c}}^{(2)}$ :

$$
\circ \Leftarrow \underbrace{\circ-\circ-\cdots-\circ-0}_{\left(N_{c}-2\right) \text { nodes }} \Leftarrow 0 .
$$

For definiteness, let us fix $\mathrm{O}^{+}$to be at $x_{3}=0$ and $\widetilde{\mathrm{O}}^{-}$ to be at $x_{3}=\pi R$. In this case, we put $N_{c} \mathrm{D} 3$ and $N_{f}-1 \mathrm{D} 5$ at $x_{3}=\frac{\pi}{2} R$. Moreover, we have one D5-brane (and its image) at $x_{3}=0$, as well as a half-physical D3-brane stuck on the $\widetilde{\mathrm{O}}^{-}$plane at $x_{3}=\pi R$.

In the dual configuration we have $N_{f}-N_{c}-1$ D3 and $N_{f}-1$ D5 at $x_{3}=\pi R$. We also have one D5-brane (and its image) at $x_{3}=0$ and a half-physical D3-brane stuck at $\widetilde{\mathrm{O3}^{-}}$ at $x_{3}=\pi R$. We can furthermore reconnect the D5-brane at $x_{3}=\frac{\pi}{2} R$, and the final configuration represents a $U\left(N_{f}-N_{c}-1\right)$ gauge theory with $N_{f}$ pairs of fundamentals and antifundamentals and superpotential $W=$ $M q \tilde{q}+t_{-}^{2}+t_{+}$.

Finally, we remark that the duality discussed in this section can also be obtained from that in Sec. IV C by a similar deformation as discussed in Sec. III C.

\section{A further argument: The $S^{3}$ partition function}

We can provide a further argument for the validity of the duality just proposed by studying the three-sphere partition function. We can indeed prove analytically the integral identity between the electric and the magnetic side. The partition function for a $U\left(N_{c}\right)$ gauge theory with $N_{f}$ pairs of fundamentals can be read from formula (A2) in the Appendix, by setting $\tau=\omega$ :

$$
Z_{U\left(N_{c}\right)}(\mu ; \nu ; \eta) \equiv Z_{U\left(N_{c}\right)}(\mu ; \nu ; \omega ; \eta) .
$$

At this point we can consider the duality between $U\left(N_{c}\right)$ with $N_{f}$ pairs of fundamentals and antifundamentals and superpotential $W=T_{+}$and $U\left(N_{f}-N_{c}-1\right)$ with $N_{f}$ pairs of fundamentals and antifundamentals and superpotential $W=M q \tilde{q}+t_{-}+t_{+} T_{-}$.

The matching between the electric and the magnetic partition functions has been proven for this case by [21]. The identity is

$$
\begin{aligned}
Z_{U\left(N_{c}\right)}(\mu ; \nu ; \eta-2 \omega)= & \mathrm{e}^{\frac{\mathrm{i} \pi}{2} \sum_{a=1}^{N_{f}}\left(\mu_{a}^{2}-\nu_{a}^{2}\right)} \Gamma_{h}(\eta) \prod_{a, b=1}^{N_{f}} \Gamma_{h}\left(\mu_{a}+\nu_{b}\right) \\
& \times Z_{U\left(N_{f}-N_{c}-1\right)}(\omega-\mu ; \omega-\nu ; \eta)
\end{aligned}
$$

where the parameters $\mu, \nu$ and $\eta$ are constrained by

$$
\frac{1}{2} \sum_{a=1}^{N_{f}}\left(\mu_{a}+\nu_{a}\right)+\frac{\eta}{2}=\omega\left(N_{f}-N_{c}\right) .
$$


Following the mathematical literature, from now on, we refer to this and similar types of identities between the parameters entering in the partition function as balancing conditions. From (4.6) one can now prove the identity for the case at hand.

This can be understood by a field theoretical analysis as follows: deforming the electric side of the duality by adding a superpotential term proportional to $T_{-}^{2}$, we impose the balancing conditions

$$
\eta=\omega, \quad \sum_{a=1}^{N_{f}}\left(\mu_{a}+\nu_{a}\right)=2 \omega\left(N_{f}-N_{c}-\frac{1}{2}\right) .
$$

By plugging (4.8) into the identity (4.6) and by using the fact that $\Gamma_{h}(\omega)=1$, one arrives at the identity

$$
\begin{aligned}
Z_{U\left(N_{c}\right)}(\mu ; \nu ;-\omega)= & \mathrm{e}^{\frac{\mathrm{i} \pi}{2} \sum_{a=1}^{N_{f}}\left(\mu_{a}^{2}-\nu_{a}^{2}\right)} \prod_{a, b=1}^{N_{f}} \Gamma_{h}\left(\mu_{a}+\nu_{b}\right) \\
& \times Z_{U\left(N_{f}-N_{c}-1\right)}(\omega-\mu ; \omega-\nu ; \omega)
\end{aligned}
$$

with the balancing conditions (4.8), which provide the equality between the partition functions that we are looking for. Furthermore, the brane picture suggests a RG flow interpolating between $\operatorname{USp}\left(2 N_{c}\right)$ with $2 N_{f}$ fundamentals and $W=Y^{2}$ and $U\left(N_{c}\right)$ with $N_{f}$ pairs of fundamentals and antifundamentals and $W=T_{+}+T_{-}^{2}$ (or $W=T_{-}+T_{+}^{2}$ ). Moreover, this flow should interpolate between the two dualities involving symplectic and unitary groups, respectively. Here, we check these expectations against the partition function. This provides a further argument in favor of the new duality for unitary theories and monopole superpotential $W=T_{+}+T_{-}^{2}\left(\right.$ or $\left.W=T_{-}+T_{+}^{2}\right)$.

\section{G. The electric flow}

The partition function for $U S p\left(2 N_{c}\right)$ with $2 N_{f}$ fundamentals can be read from formula (A3) in the Appendix by setting $\tau=\omega$. We have

$$
Z_{U S p\left(2 N_{c}\right)}(m) \equiv Z_{U S p\left(2 N_{c}\right)}(m ; \omega) .
$$

On the electric side we then consider the partition function $Z_{\text {ele }}=Z_{U S p\left(2 N_{c}\right)}(m)$. The quadratic monopole superpotential imposes the balancing condition

$$
\sum_{a=1}^{2 N_{f}} m_{a}=\omega\left(2 N_{f}-2 N_{c}-1\right) .
$$

We then consider the Higgs flow triggered by the shift ${ }^{3}$

\footnotetext{
${ }^{3} \mathrm{We}$ could have chosen the opposite signs for $s$. This choice corresponds to $W=T_{+}+T_{-}^{2}$, while the opposite choice corresponds to $W=T_{-}+T_{+}^{2}$
}

$$
\sigma_{a} \rightarrow \sigma_{a}-s, \quad a=1, \ldots, N_{c}
$$

and the real mass flow triggered by

$m_{i} \rightarrow \mu_{i}+s, \quad m_{i+N_{f}} \rightarrow \nu_{i}-s \quad i=1, \ldots, N_{f}$.

By plugging (4.17) and (4.13) into $Z_{U S p\left(2 N_{c}\right)}(m)$ and by computing the large $s$ limit using formula (A4), we arrive at the partition function of the $U\left(N_{c}\right)$ gauge theory with $N_{f}$ pairs of fundamentals and antifundamentals and $W=T_{+}+T_{-}^{2}$,

$$
Z_{\text {ele }}=\frac{\mathrm{e}^{\frac{\mathrm{i} \pi}{2}(A s+B)}}{N_{c} !} Z_{U\left(N_{c}\right)}(\mu ; \nu ;-\omega)
$$

where

$$
\begin{aligned}
& A=-4 N_{c}^{2} \omega, \\
& B=N_{c}\left(\sum_{i=1}^{N_{f}}\left(\mu_{i}^{2}-\nu_{i}^{2}\right)-2 \omega \sum_{i=1}^{N_{f}}\left(\mu_{i}-\nu_{i}\right)\right) .
\end{aligned}
$$

\section{H. The magnetic flow}

On the magnetic side the partition function is

$$
Z_{\mathrm{mag}}=\prod_{1 \leq a<b \leq 2 N_{f}} \Gamma_{h}\left(m_{i}+m_{j}\right) Z_{U S p\left(2 \tilde{N}_{c}\right)}\left(\tilde{m}_{i}\right)
$$

with $\tilde{N}_{c}=N_{f}-N_{c}-1$ and $\tilde{m}_{j}=\omega-m_{j}$. The dual Higgs flow is triggered by

$$
\sigma_{a} \rightarrow \sigma_{a}+s, \quad a=1, \ldots, \tilde{N}_{c}
$$

while the real mass flow can be read by using the duality map from the electric one. In the large $s$ limit we arrive at the partition function of the $U\left(\tilde{N}_{c}\right)$ gauge theory with $N_{f}$ pairs of fundamentals and antifundamentals, $N_{f}^{2}$ singlets $M_{i j}$ (with $i, j=1, \ldots, N_{f}$ ) and $W=M q \tilde{q}+t_{-}+t_{+}^{2}$,

$$
Z_{\text {mag }}=\mathrm{e}^{\tilde{A} s+\tilde{B}} \prod_{i, j=1}^{N_{f}} \Gamma_{h}\left(\mu_{i}+\nu_{j}\right) Z_{U\left(\tilde{N} \tilde{N}_{c}\right)}(\tilde{\mu}, \tilde{\nu} ; \omega)
$$

where $\tilde{\mu}=\omega-\mu, \tilde{\nu}=\omega-\nu$ and

$\tilde{A}=A$,

$\tilde{B}=\left(N_{c}-1\right) \sum_{i=1}^{N_{f}}\left(\mu_{i}^{2}-\nu_{i}^{2}\right)-\omega\left(2 N_{c}-1\right) \sum_{i=1}^{N_{f}}\left(\mu_{i}-\nu_{i}\right)$.

Moreover, using the fact that $\sum \mu_{i}=\sum \nu_{i}$ we can equate (4.14) and (4.18), and we are left with the identity (4.9) as expected. 


\section{DUALITIES WITH TENSORIAL MATTER}

In this section we study $3 \mathrm{~d} \mathcal{N}=2$ dualities in the presence of tensorial matter fields and a quadratic monopole superpotential. The analysis is inspired from the discussion in Sec. 4.1.1 in [37] and here in (IV F 1). The idea consists in deforming an electric duality by a quadratic monopole superpotential and finding the dual deformation on the magnetic side. These superpotentials impose a set of constraints on the complex combinations of real masses and $R$-charges appearing as parameters in the partition function. After fixing these constraints, the identities relating the partition functions of the parent dualities become new identities among the partition functions of the new dualities. In this last step some singlets may disappear from the identity because they contribute as fields with holomorphic masses; i.e., their partition function is equal to one.

\section{A. $U\left(N_{c}\right)$ gauge group}

We start our analysis by considering SQCD with $U\left(N_{c}\right)$ gauge groups, $N_{f}$ fundamentals $Q$ and antifundamentals $\tilde{Q}$, and one adjoint matter field with superpotential

$$
W_{\text {ele }}=\operatorname{tr} X^{k+1} .
$$

This theory is dual to $U\left(k N_{f}-N_{c}\right)$ SQCD with $N_{f}$ dual fundamentals $q$ and antifundamentals $\tilde{q}, k$ mesons $M_{j}=Q X^{j} \tilde{Q}, j=0, \ldots, k-1$, and an adjoint $Y$ with superpotential

$$
\begin{aligned}
W_{\mathrm{mag}}= & \operatorname{tr} Y^{k+1}+\sum_{j=0}^{k-1} M_{j} q Y^{k-1-j} \tilde{q} \\
& +\sum_{j=0}^{k-1}\left(T_{j} t_{k-1-j}+\tilde{T}_{j} \tilde{t}_{k-1-j}\right)
\end{aligned}
$$

where $T_{j}=T_{0} \operatorname{tr} X^{j}, \tilde{T}_{j}=\tilde{T}_{0} \operatorname{tr} X^{j}, t_{j}=t_{0} \operatorname{tr} Y^{j}$, and $\tilde{t}_{j}=\tilde{t}_{0} \operatorname{tr} Y^{j}$, and $T_{0}, \tilde{T}_{0}, t_{0}$ and $\tilde{t}_{0}$ are the bare monopoles and antimonopoles of the electric and of the magnetic theory, respectively. This duality, known as Kim-Park duality [39], can be modified into a duality involving quadratic monopoles. There are two possibilities, depending on $k$ being even or odd.

(i) For even $k$ we add to the electric theory the monopole superpotential

$$
\Delta W_{\text {ele }}=T_{\frac{k}{2}}^{2}+\tilde{T}_{\frac{k}{2}-1}^{2},
$$

or equivalently

$$
\Delta W_{\text {ele }}=T_{\frac{k}{2}-1}^{2}+\tilde{T}_{\frac{k}{2}}^{2} .
$$

This corresponds to adding the following superpotential to the magnetic theory:

$$
\Delta W_{\mathrm{mag}}=t_{\frac{k}{2}}^{2}+\tilde{t}_{\frac{k}{2}-1}^{2}
$$

or equivalently

$$
\Delta W_{\mathrm{mag}}=t_{\frac{k}{2}-1}^{2}+\tilde{t}_{\frac{k}{2}}^{2} .
$$

(ii) For odd $k$ we add to the electric theory the monopole superpotential

$$
\Delta W_{\text {ele }}=T_{\frac{k-1}{2}}^{2}+\tilde{T}_{\frac{k-1}{2}}^{2} .
$$

This corresponds to adding the following superpotential to the magnetic theory:

$$
\Delta W_{\mathrm{mag}}=t_{\frac{k-1}{2}}^{2}+\tilde{t}_{\frac{k-1}{2}}^{2} .
$$

From now on we discuss only the case of odd $k$ and then comment on the other case at the end. By adding the superpotential (5.7) we constrain the $R$-charges of the monopoles and, as a consequence, the one of the matter fields. We are left with the constraint

$$
N_{f}(1-\Delta)-\left(N_{c}-1-\frac{k-1}{2}\right) \frac{2}{k+1}=1
$$

where $R[Q]=R[\tilde{Q}]=\Delta$. Observe that if we add the same superpotential in the dual theory, the constraint is

$$
N_{f}\left[1-\left(\frac{2}{k+1}-\Delta\right)\right]-\left(\tilde{N}_{c}-1-\frac{k-1}{2}\right) \frac{2}{k+1}=1
$$

and, at the level of the charges, this is consistent with the duality only if $\tilde{N}_{c}=k N_{f}-N_{c}$. This fact can be confirmed by looking at the partition function. The identity for the Kim-Park duality is

$$
\begin{aligned}
& Z_{U\left(N_{c}\right)}(\mu ; \nu ; \tau ; \eta) \\
& =\prod_{j=0}^{k-1} \Gamma_{h}\left( \pm \frac{\eta}{2}+\omega N_{f}+\left(j-N_{c}+1\right) \tau-\sum_{a=1}^{N_{f}} \frac{\mu_{a}+\nu_{a}}{2}\right) \\
& \quad \times \prod_{a, b=1}^{N_{f}} \Gamma_{h}\left(j \tau+\mu_{a}+\nu_{b}\right) Z_{U\left(\tilde{N}_{c}\right)}(\tilde{\mu} ; \tilde{\nu} ; \tau ;-\eta)
\end{aligned}
$$

where we refer to the Appendix for the various notations.

By adding the superpotential (5.7) we introduce a balancing condition

$$
\omega N_{f}+\left(\frac{k-1}{2}-N_{c}+1\right) \tau-\sum_{a=1}^{N_{f}} \frac{\mu_{a}+\nu_{a}}{2}=\omega .
$$

Since 


$$
\tau=\omega \Delta_{A}=\frac{2}{k+1} \omega
$$

this simplifies to

$$
\sum_{a=1}^{N_{f}}\left(\mu_{a}+\nu_{a}\right)=2\left(\omega N_{f}-\tau N_{c}\right) .
$$

Furthermore, we set $\eta=0$. In each of the first and second lines of (5.11), the terms in the product can be paired between $j=m$ and $j=(k-1)-m$, with $0 \leq m \leq k-1$. In each of these two lines, there is also an unpaired term for $j=\frac{1}{2}(k-1)$. Using the identity $\Gamma_{h}(2 \omega-x) \Gamma_{h}(x)=1$, it can be seen that the contributions of each pair cancel precisely, and the unpaired term gives $\Gamma_{h}(\omega)=1$. Hence, we have proven that

$$
\begin{aligned}
& Z_{U\left(N_{c}\right)}(\mu ; \nu ; \tau ; 0) \\
& \quad=\prod_{a, b=1}^{N_{f}} \Gamma_{h}\left(j \tau+\mu_{a}+\nu_{b}\right) Z_{U\left(\tilde{N}_{c}\right)}(\tilde{\mu} ; \tilde{\nu} ; \tau ; 0)
\end{aligned}
$$

with the duality map $\tilde{\mu}=\tau-\mu$ and $\tilde{\nu}=\tau-\nu$ and the balancing condition (5.14). Observe that the even cases work in a similar manner, essentially because they leave the balancing condition (5.14) unchanged.

\section{More general monopole superpotentials}

The above discussion can be generalized in the case in which we add, to the electric theory, the monopole superpotential

$$
\Delta W_{\text {ele }}=T_{q}^{2}+\tilde{T}_{k-1-q}^{2}
$$

and similarly, to the magnetic theory, the monopole superpotential

$$
\Delta W_{\mathrm{mag}}=t_{q}^{2}+\tilde{t}_{k-1-q}^{2}
$$

Let us first analyze the electric theory. It can be easily seen that the basic monopole operators $T_{0}$ and $\tilde{T}_{0}$ have different $R$-charges if $q \neq(k-1) / 2$. Moreover, the $U(1)_{T}$ topological symmetry and the $U(1)_{R} R$-symmetry are broken to a diagonal subgroup. Let us refer to the latter as $U(1)_{R^{\prime}}=U(1)_{R}-\alpha U(1)_{T}$. Therefore,

$$
R^{\prime}\left[T_{0}\right]=R-\alpha, \quad R^{\prime}\left[\tilde{T}_{0}\right]=R+\alpha,
$$

with

$$
\begin{aligned}
R & =N_{f}(1-\Delta)+\left(N_{c}-1\right)\left(1-\Delta_{A}\right)-\left(N_{c}-1\right), \\
\Delta_{A} & =\frac{2}{k+1} .
\end{aligned}
$$

The $R^{\prime}$-charges of $T_{q}$ and $\tilde{T}_{k-1-q}$ can be written as follows:

$$
\begin{aligned}
R^{\prime}\left[T_{q}\right] & =1=R-\alpha+q \Delta_{A}, \\
R^{\prime}\left[\tilde{T}_{k-1-q}\right] & =1=R+\alpha+(k-1-q) \Delta_{A} .
\end{aligned}
$$

Solving these equations yields

$$
\alpha=(q+1) \Delta_{A}-1=\frac{2 q-(k-1)}{k+1} .
$$

This is in agreement with the above analyses for $q=$ $(k-1) / 2$ with $k$ odd, and for $q=k / 2$ with $k$ even.

Similarly for the magnetic theory, we have

$$
R^{\prime}\left[t_{0}\right]=\hat{R}-\alpha, \quad R^{\prime}\left[\tilde{t}_{0}\right]=\hat{R}+\alpha,
$$

with

$$
\begin{aligned}
\hat{R}= & N_{f}\left[1-\left(\Delta_{A}-\Delta\right)\right]+\left(\tilde{N}_{c}-1\right)\left(1-\Delta_{A}\right) \\
& -\left(\tilde{N}_{c}-1\right),
\end{aligned}
$$

and

$$
\begin{aligned}
R^{\prime}\left[t_{q}\right] & =1=\hat{R}-\alpha+q \Delta_{A}, \\
R^{\prime}\left[\tilde{t}_{k-1-q}\right] & =1=\hat{R}+\alpha+(k-1-q) \Delta_{A} .
\end{aligned}
$$

Solving these equations, we obtain

$$
\tilde{N}_{c}=k N_{f}-N_{c} .
$$

We see that the sum of the equations in (5.20) gives rise to the same balancing condition as (5.14), which is independent of $q$. It should be emphasized that for $q \neq \frac{k-1}{2}$, the FI parameter $\xi$ in (5.11) can be nonzero (in contrast to $q=\frac{k-1}{2}$ ). In this case, we can pair the terms $j=m$ in the first line with $j=(k-1)-m$ in the second line, for $0 \leq m \leq k-1$. Upon using the identity $\Gamma_{h}(2 \omega-x) \Gamma_{h}(x)=1$, we see that the contribution from each pair cancels precisely. We thus arrive at a similar relation to (5.15):

$$
\begin{aligned}
& Z_{U\left(N_{c}\right)}(\mu ; \nu ; \tau ; \eta) \\
& \quad=\prod_{a, b=1}^{N_{f}} \Gamma_{h}\left(j \tau+\mu_{a}+\nu_{b}\right) Z_{U\left(\tilde{N}_{c}\right)}(\tilde{\mu} ; \tilde{\nu} ; \tau-\eta) .
\end{aligned}
$$

Thus, the same duality holds with the addition of (5.16) and (5.17) for any $0 \leq q \leq k-1$, with a nonzero FI parameter in the partition function. 


\section{B. $U\left(N_{c}\right)$ with a single quadratic monopole superpotential}

Here we discuss a duality between

(i) $U\left(N_{c}\right)$ adjoint SQCD with

$$
W=X^{k+1}+\tilde{T}_{\frac{k-1}{2}}^{2}
$$

and $k$ even, and

(ii) $U\left(k N_{f}-N_{c}\right)$ adjoint SQCD with

$$
W=Y^{k+1}+t_{\frac{k-1}{2}}^{2}+\sum_{j=0}^{k-1} M_{j} q Y^{k-1-j} \tilde{q}+\sum_{j=1}^{k-1} T_{j} \tilde{t}_{k-1-j} .
$$

Observe that a more general duality can be constructed by considering a monopole superpotential $W \sim \tilde{T}_{q}^{2}$ with $0 \leq q \leq k-1$. Such a duality can be defined for both even and odd $k$, and it just requires more care in the choice of the FI [see the discussion at the end of Sec. (VA)]. We will not discuss this generalization further, and we leave the details to the interested reader.

Here we show that the duality for even $k$ summarized above can be obtained from the duality with a quadratic monopole superpotential discussed in Sec. (VA). We consider the case with $N_{f}+1$ fundamentals and trigger a real mass flow on the partition function by considering the large $s$ limit in the relations

$$
\mu_{N_{f}+1} \rightarrow \frac{\eta}{2}+s, \quad \nu_{N_{f}+1} \rightarrow \frac{\eta}{2}-s .
$$

The balancing condition (5.14) is modified as

$$
\sum_{a=1}^{N_{f}}\left(\mu_{a}+\nu_{a}\right)+\eta+2 N_{c} \tau=2 \omega\left(N_{f}+1\right) .
$$

In the dual side we further consider the Higgs flow in the gauge sector, breaking the gauge symmetry as $U\left(k\left(N_{f}+1\right)-N_{c}\right) \rightarrow U\left(k N_{f}-N_{c}\right) \times U(k)$.

By performing the large $s$ limit in the identity (5.15), we are left with the identity between two finite quantities, after we simplify the divergent pieces. The subsequent analysis is very similar to that presented in Sec. 4.1.1 of [37].

On the electric side we have the partition function of $U\left(N_{c}\right)$ adjoint SQCD with superpotential (5.39) and effective FI equal to $\left(\frac{\eta}{2}-\omega\right)$. The presence of the quadratic monopole superpotential in (5.39) is captured by the balancing condition (5.30). On the magnetic side we have two gauge sectors. The first one corresponds to $U\left(k N_{f}-N_{c}\right)$ adjoint SQCD, and it captures the first three terms in the superpotential (5.40). The last term in (5.40) (i.e., the contribution of the electric dressed monopoles $\tilde{T}_{j}$ acting as singlets in the dual phase) is captured by the second integral. In addition there are $j$ contributions from the $\left(N_{f}+1\right)$ th components of the original dressed mesons, which are massless in this dual phase, after triggering the real mass flow as in (5.29). The contribution of the singlets $T_{j}$ can be seen explicitly by studying the partition function associated to this extra gauge sector and these $j$ singlets arising from the original meson. We have

$$
\begin{aligned}
\prod_{j=0}^{k-1} & \Gamma_{h}(\eta+j \tau) \int \prod_{c=1}^{k} \mathrm{~d} \sigma_{c} \mathrm{e}^{\mathrm{i} \pi(\eta-2 \omega)} \Gamma_{h}\left(\tau-\frac{\eta}{2} \pm \sigma_{c}\right) \\
& \times \prod_{c<d} \frac{\Gamma_{h}\left( \pm\left(\sigma_{c}-\sigma_{d}\right)+\tau\right)}{\Gamma_{h}\left( \pm\left(\sigma_{c}-\sigma_{d}\right)\right)} \\
= & \prod_{j=0}^{k-1} \Gamma_{h}(\eta+j \tau) \Gamma_{h}(\eta-(j+1) \tau) \Gamma_{h}(2 \omega-(j+1) \tau) \\
& \times \Gamma_{h}((2-j) \tau-\eta) \\
= & \prod_{j=0}^{k-1} \Gamma_{h}(\eta-(j+1) \tau)=\prod_{j=0}^{k-1} \Gamma_{h}(\eta-(k-j) \tau)
\end{aligned}
$$

where we have evaluated this integral by using Theorem 5.6.8 of [31]. We can show that (5.31) corresponds to the electric monopole by applying the balancing condition (5.30):

$$
\begin{aligned}
\eta-(k-j) \tau= & \left(\frac{\eta}{2}-\omega\right)+\left[j-k-N_{c}\right] \tau+\omega\left(N_{f}+2\right) \\
& -\frac{1}{2} \sum_{a=1}^{N_{f}}\left(\mu_{a}+\nu_{a}\right) .
\end{aligned}
$$

In order to see that this combination captures the global charges of the dressed monopoles $T_{j}$ acting as singlets in the last sum of the superpotential (5.40), we have to shift the effective FI as $\eta \rightarrow \eta+2 \omega$. In this way the FI is chosen canonically, and we can simply read the global charges from the combination of the real masses in (5.32). After the shift and some rearranging, the rhs of (5.32) becomes

$$
\frac{\eta}{2}+\omega N_{f}-\left(N_{c}-j-1\right) \tau-\frac{1}{2} \sum_{a=1}^{N_{f}}\left(\mu_{a}+\nu_{a}\right) .
$$

From this relation we can see that this field has topological charge +1 , axial mass $-N_{f}$ and $R$-charge

$$
\Delta_{j}=N_{f}\left(1-\Delta_{Q}\right)-\Delta_{X}\left(N_{c}-j-1\right)
$$

where $\Delta_{Q}$ are the charges of the electric fundamentals $Q$ and antifundamentals $\tilde{Q}$ (with $\Delta_{Q}=\Delta_{\tilde{Q}}$ ) and $\Delta_{X}$ is the $R$-charge of the electric adjoint field $X$. This shows that the expression in (5.33) is the combination of masses and charges expected for the (dressed) electric monopoles.

Observe that from this duality we can further flow to the identity (5.11) by triggering a further real mass flow. 
This provides a further consistency check of the duality. We leave the details of this calculation to the interested reader.

\section{C. $U\left(N_{c}\right)_{\mathcal{K}}$ with quadratic monopole superpotentials}

It is also possible to study a RG flow leading to a duality involving CS matter theories. This is done by turning on the real masses for the fundamentals and shifting the scalars $\sigma_{i}$ and the FI as

$$
\begin{aligned}
\mu_{a} & \rightarrow \mu_{a}-\kappa s & & a=1, \ldots, N_{f}-\kappa \\
\mu_{a} & \rightarrow \mu_{a}+\left(2 N_{f}-\kappa\right) s & & a=N_{f}-\kappa+1, \ldots, N_{f} \\
\nu_{a} & \rightarrow \nu_{a}+\kappa s & & a=1, \ldots, N_{f} \\
\eta & \rightarrow \eta-2 N_{f} \kappa s & & \\
\sigma_{i} & \rightarrow \sigma_{i}+\kappa s & & i=1, \ldots, N_{c} \\
\tilde{\sigma}_{i} & \rightarrow \sigma_{i}-\kappa s & & i=1, \ldots, \tilde{N}_{c}
\end{aligned}
$$

where $\tilde{\sigma}_{i}$ is the shift of the scalar in the dual vector multiplet. The real masses in the dual theory can be read from the duality map as usual. We can study the real mass flow by computing the large $s$ limit on the partition function. We find the following identity:

$$
\begin{aligned}
Z_{U\left(N_{c}\right) \frac{\kappa}{2}}\left(\mu ; \nu ; \tau ; \eta_{\text {ele }}\right)= & \mathrm{e}^{\frac{\mathrm{i} \pi}{2} \phi} \prod_{a=1}^{N_{f}-\kappa} \prod_{b=1}^{N_{f}} \prod_{j=0}^{k-1} \Gamma_{h}\left(\mu_{a}+\nu_{b}+j \tau\right) \\
& \times Z_{U\left(k N_{f}-N_{c}\right)_{-\frac{\kappa}{2}}}\left(\tau-\mu ; \tau-\nu ; \tau ; \eta_{\text {mag }}\right)
\end{aligned}
$$

where the electric and the magnetic FI in (5.36) are

$$
\begin{gathered}
\eta_{\mathrm{ele}}=-2\left(\sum_{a=1}^{N_{f}-\kappa} \mu_{a}-\sum_{b=1}^{N_{f}} \nu_{b}+\eta-\omega(\kappa+2)\right), \\
\eta_{\mathrm{mag}}=-2\left(\sum_{a=1}^{N_{f}-\kappa} \mu_{a}-\sum_{b=1}^{N_{f}} \nu_{b}+\eta-\kappa \tau+\omega(\kappa-2)\right)
\end{gathered}
$$

and the phase $\phi$ in (5.36) is

$$
\begin{aligned}
\phi= & k\left(\kappa \sum_{b=1}^{N_{f}} \nu_{b}^{2}-2\left(\sum_{b=1}^{N_{f}} \nu_{b}-\sum_{a=1}^{N_{f}-\kappa} \mu_{a}\right)\right. \\
& \left.\times\left(\sum_{b=1}^{N_{f}} \nu_{b}+\tau N_{c}-\omega N_{f}\right)-2 \kappa \tau \sum_{b=1}^{N_{f}} \nu_{b}\right) \\
& -\frac{1}{3} k\left(\kappa \tau\left(3 \tau N_{c}+(k-4) \omega N_{f}\right)-\tau \omega+13 \omega^{2}\right) \\
& -k \eta^{2}+4 \eta k \omega .
\end{aligned}
$$

This is compatible with a duality between

(i) $U\left(N_{c}\right)_{\frac{\kappa}{2}}$ adjoint SQCD with $N_{f}-\kappa$ fundamentals and $N_{f}^{2}$ antifundamentals and superpotential

$$
W=X^{k+1}+\tilde{T}_{\frac{k-1}{2}}^{2}
$$

and $k$ even, and

(ii) $U\left(k N_{f}-N_{c}\right)_{-\frac{k}{2}}$ adjoint SQCD with $N_{f}-\kappa$ fundamentals and $N_{f}^{2}$ antifundamentals and superpotential

$$
W=Y^{k+1}+t_{\frac{k-1}{2}}^{2}+\sum_{j=0}^{k-1} M_{j} q Y^{k-1-j} \tilde{q} .
$$

This duality generalizes that of Sec. 8.1 of [21] for the linear monopole superpotential and that of Sec. 3.2.3 of [37] for the quadratic monopole superpotential.

\section{D. $U\left(N_{c}\right)$ with linear and quadratic monopole superpotentials}

Here we discuss a duality between

(i) $U\left(N_{c}\right)$ adjoint SQCD with

$$
W=X^{k+1}+\tilde{T}_{\frac{k-1}{2}}^{2}+T_{0}
$$

and $k$ even, and

(ii) $U\left(k\left(N_{f}-1\right)-N_{c}\right)$ adjoint SQCD with

$$
W=Y^{k+1}+\sum_{j=0}^{k-1} M_{j} q Y^{k-1-j} \tilde{q}+\tilde{t}_{\frac{k-1}{2}}^{2}+t_{0} .
$$

Again a more general duality can be constructed, including also the $k$ odd case, by considering a monopole superpotential $W \sim \tilde{T}_{q}^{2}$ (or $W \sim T_{q}^{2}$ ) with $0 \leq q \leq k-1$. We will not further discuss this generalization here.

Here we provide evidence of this duality, showing that it can be obtained from a duality discussed in [17] involving $U\left(N_{c}\right)$ adjoint SQCD with $W=X^{k+1}+T_{0}$ and $U\left(k\left(N_{f}-1\right)-N_{c}\right)$ adjoint SQCD with $W=Y^{k+1}+$ $\sum_{j=1}^{k-1} M_{j} q Y^{k-1-j} \tilde{q}+t_{0}$.

Our argument will be based on the matching of the partition functions. We start from the relation derived in [17],

$$
\begin{aligned}
& Z_{U\left(N_{c}\right)}(\mu ; \nu ; \tau ; \omega-\eta) \\
& =\prod_{j=0}^{k-1} \Gamma_{h}(2 \eta+\tau j) \prod_{a, b=1}^{N_{f}} \Gamma_{h}\left(\mu_{a}+\nu_{b}+j \tau\right) \\
& \quad \times Z_{U\left(k\left(N_{f}-1\right)-N_{c}\right)}(\tau-\mu ; \tau-\nu ; \tau ; \tau-\omega-\eta) .
\end{aligned}
$$

Note that in the $U\left(N_{c}\right)$ theory the FI parameter is taken to be $\omega-\eta$. This identity is valid provided the condition

$$
\sum_{a=1}^{N_{f}}\left(\mu_{a}+\nu_{a}\right)+\eta=\omega\left(N_{f}-1\right)-\tau\left(N_{c}-1\right)
$$


on the parameters is imposed. Next we add the quadratic superpotential $W \sim \tilde{T}_{\frac{k-1}{2}}^{2}$ on the electric side of the duality. It corresponds to fixing $\eta=\frac{\tau}{2}$, due to the fact that

$$
\begin{aligned}
1 & =R\left[\tilde{T}_{\frac{k-1}{2}}\right]=R\left[\tilde{T}_{0}\right]+\left(\frac{k-1}{2}\right) \Delta_{A} \\
& =R\left[\tilde{T}_{0}\right]+\left(1-\Delta_{A}\right) .
\end{aligned}
$$

On the magnetic side the effect of this deformation can be argued by looking at the partition function (5.43). The net effect consists of giving a holomorphic mass to the singlets associated to the monopoles of the electric theory:

$$
\begin{aligned}
\prod_{j=0}^{k-1} \Gamma_{h}(2 \eta+\tau j) & =\prod_{j=0}^{k-1} \Gamma_{h}(\tau(j+1)) \\
& =\Gamma_{h}(\tau) \ldots \Gamma_{h}(k \tau)=1 .
\end{aligned}
$$

We are then left with the identity

$$
\begin{aligned}
& Z_{U\left(N_{c}\right)}\left(\mu ; \nu ; \tau ; \omega-\frac{\tau}{2}\right) \\
& =\prod_{j=0}^{k-1} \prod_{a, b=1}^{N_{f}} \Gamma_{h}\left(\mu_{a}+\nu_{b}+j \tau\right) \\
& \quad \times Z_{U\left(k\left(N_{f}-1\right)-N_{c}\right)}\left(\tau-\mu ; \tau-\nu ; \tau ; \frac{\tau}{2}-\omega\right)
\end{aligned}
$$

with the balancing condition

$$
\sum_{a=1}^{N_{f}}\left(\mu_{a}+\nu_{a}\right)=\omega\left(N_{f}-1\right)-\tau\left(N_{c}-\frac{1}{2}\right) .
$$

The relation (5.47) together with the balancing condition (5.48) represents the matching of the partition function for the duality summarized at the beginning of this subsection. Observe that the presence of the quadratic monopole in the magnetic superpotential, $W \sim \tilde{t}_{\frac{k-1}{2}}^{2}$, can be argued because it is consistent with the constraints on the global charges given by (5.48).

\section{E. $U S p\left(2 N_{c}\right)$ gauge group}

The above duality can easily be generalized to theories with symplectic gauge groups. We propose the duality between the following theories:

\section{Theory A}

The $\operatorname{USp}\left(2 N_{c}\right)$ gauge theory with $2 N_{f}$ fundamentals $Q^{a}$, an antisymmetric traceless chiral multiplet $A$, and a superpotential

$$
W=\operatorname{tr} A^{k+1}+T_{q}^{2},
$$

where $T_{q}$ is the dressed monopole operator

$$
T_{q}=Y \operatorname{tr}\left(A^{q}\right)
$$

with $q$ an integer and $Y$ the basic monopole operator of theory A.

\section{Theory $B$}

The $\operatorname{USp}\left(2 \tilde{N}_{c}\right)$ gauge theory with $2 N_{f}$ fundamentals, an antisymmetric traceless chiral multiplet $a$, singlets $M_{j}=Q^{a} Y^{j} Q^{b}(j=0, \ldots, 2 k)$, singlets $T_{j}=T_{0} \operatorname{tr} X^{j}$, and a superpotential

$$
W=\operatorname{tr} a^{k+1}+\sum_{j=0}^{k-1} M_{k-j-1} q a^{j} q+t_{q}^{2}
$$

where $t_{q}$ is the dressed monopole operator

$$
t_{q}=\tilde{Y} \operatorname{tr}\left(a^{q}\right)
$$

with $\tilde{Y}$ the basic monopole operator of theory B.

We see that the duality holds provided that

$$
\tilde{N}_{c}=\left(N_{f}-1\right) k-N_{c}, \quad q=\frac{1}{2}(k-1) .
$$

In order for $q$ to be an integer, $k$ has to be odd. However, if $k$ is even, $q$ is half-odd-integral, and we need to redefine the dressed monopole operators $T_{q}$ and $t_{q}$. One possibility is to define $^{4}$

$$
T_{q}=Y(\operatorname{det} A)^{\frac{q}{2 N_{c}}}, \quad t_{q}=\tilde{Y}(\operatorname{det} a)^{\frac{q}{2 N_{c}}}
$$

for $q$ either integral or half-odd-integral.

From the superpotentials, we see that the $R$-charges of $A$ and $a$ are equal to

$$
\Delta_{A}=\frac{2}{k+1} .
$$

In order to see the first equality of (5.53), we consider the $R$-charges of the monopole operators $T_{q}$ and $t_{q}$ :

$$
\begin{aligned}
1=R\left[T_{q}\right]= & 2 N_{f}(1-r)+q \Delta_{A}+\left(1-\Delta_{A}\right) 2\left(N_{c}-1\right)-2 N_{c} \\
1=R\left[t_{q}\right]= & 2 N_{f}\left(1-\left(\Delta_{A}-r\right)\right)+q \Delta_{A} \\
& +\left(1-\Delta_{A}\right) 2\left(\tilde{N}_{c}-1\right)-2 \tilde{N}_{c} .
\end{aligned}
$$

\footnotetext{
${ }^{4}$ The determinant of $A$ is related to the trace of a power of $A$ by Newton's identities. Note that since $A$ is an antisymmetric matrix, the trace of an odd power of $A$ is zero. Thus, for example, if $A$ is a two-by-two matrix, we have $\operatorname{det} A=-\frac{1}{2} \operatorname{tr}\left(A^{2}\right)$; and if $A$ is a fourby-four matrix, we have $\operatorname{det} A=\frac{1}{8}\left(\operatorname{tr}\left(A^{2}\right)\right)^{2}-\frac{1}{4} \operatorname{tr}\left(A^{4}\right)$.
} 
Solving these equations, we obtain

$$
\tilde{N}_{c}=\left(N_{f}-1\right) k-N_{c}+\left[q-\frac{1}{2}(k-1)\right] .
$$

The monopole superpotential in theory $A$ gives rise to the balancing condition:

$$
2 \omega N_{f}+\left[q-2\left(N_{c}-1\right)\right] \tau-\sum_{a=1}^{2 N_{f}} \mu_{a}=3 \omega .
$$

The identity for the duality without monopole superpotentials is given by [see Eq. (5.5) of [6] ]

$$
\begin{aligned}
& Z_{U S p\left(2 N_{c}\right)}(\mu ; \tau) \\
& =\prod_{j=0}^{k-1} \prod_{a<b} \Gamma_{h}\left(\mu_{a}+\mu_{b}+j \tau\right) Z_{U S p\left(2 \tilde{N}_{c}\right)}(\tau-\mu ; \tau) \\
& \quad \times \prod_{j=0}^{k-1} \Gamma_{h}\left(-2 \omega+2 \omega N_{f}+\left[j-2\left(N_{c}-1\right)\right] \tau-\sum_{a=1}^{2 N_{f}} \mu_{a}\right) .
\end{aligned}
$$

Let us assume that $k$ is odd. We see that the terms in the product in the second line of the above equation can be paired between $j=m$ and $j=(k-1)-m$, with $0 \leq m \leq k-1$. There is an unpaired term for $j=$ $\frac{1}{2}(k-1)$. The argument of $\Gamma_{h}$ for each pair adds up to

$-4 \omega+4 \omega N_{f}+2\left[\frac{1}{2}(k-1)-2\left(N_{c}-1\right)\right] \tau-2 \sum_{a=1}^{2 N_{f}} \mu_{a}$.

Upon using the balancing condition (5.58), with $q=$ $\frac{1}{2}(k-1)$, the above expression becomes $2 \omega$. We can then use the identity $\Gamma_{h}(2 \omega-x) \Gamma(x)=1$ to cancel the contribution of each pair. On the other hand, the argument of $\Gamma_{h}$ for the unpaired term is

$$
-2 \omega+2 \omega N_{f}+\left[\frac{1}{2}(k-1)-2\left(N_{c}-1\right)\right] \tau-\sum_{a=1}^{2 N_{f}} \mu_{a}=\omega,
$$

where we have used again the balancing condition (5.58), with $q=\frac{1}{2}(k-1)$. Since $\Gamma_{h}(\omega)=1$, we obtain

$$
\begin{aligned}
Z_{U S p\left(2 N_{c}\right)}(\mu ; \tau) & =\prod_{j=0}^{k-1} \prod_{a<b} \Gamma_{h}\left(\mu_{a}+\mu_{b}+j \tau\right) Z_{U S p\left(2 \tilde{N}_{c}\right)}(\tau-\mu ; \tau), \\
q & =\frac{1}{2}(k-1) .
\end{aligned}
$$

We thus establish the duality between theories $A$ and $B$, with the parameters $q=\frac{1}{2}(k-1)$ and thus $\tilde{N}_{c}=\left(N_{f}-1\right) k-N_{c}$, along with the duality map $\tilde{\mu}=\tau-\mu, \tilde{\nu}=\tau-\nu$.

In the case in which $k$ is even, we see that there is no unpaired term and the contributions from each pair cancel precisely. However, in this case, $q$ takes a half-odd-integral value, so the dressed monopole operators have to be redefined as, for example, in (5.54).

\section{CONCLUSIONS}

In this paper we discussed $3 \mathrm{~d} \mathcal{N}=2$ dualities in the presence of quadratic monopole superpotentials. In the first part of the paper we provided a brane picture of such dualities for SQCD with symplectic and unitary gauge groups. The basic observation is that these dualities can be obtained by $T$-duality on the $4 \mathrm{~d}$ picture in the presence of orientifolds, as discussed in [8-11]. The new ingredient that allowed us to generalize the construction to the cases with quadratic monopole superpotentials corresponds to also considering twisted affine compactifications. The twist is due to an outer automorphism of the gauge algebra, and it implies that after $T$-duality we can have all the possible pairs involving $\mathrm{O3}^{-}, \mathrm{O}^{+}$and $\widetilde{\mathrm{O3}^{-}}$, acting on the compact direction. This provides a classification scheme for the $3 \mathrm{~d}$ $\mathcal{N}=2$. In this way we also obtain a new duality for the unitary case, with a linear and a quadratic monopole superpotential. This duality has been checked against the partition function as well. In the second part of the paper we used similar arguments on the partition function to construct new dual pairs for dualities with tensorial matter, adjoint for the unitary case and antisymmetric for the symplectic one.

In the analysis we left some open question on which we would like to come back in the future. First, we did not discuss the orthogonal case. This corresponds to considering the gauge theory living on $\mathrm{O}^{-}$and on $\widetilde{\mathrm{O}}^{-}$planes. The monopole superpotential in these cases has a more intricate structure because it involves the monopole $Y_{\text {Spin }}$ or the monopole $Y \propto \mathrm{e}^{\Sigma_{1}}$. The two are related as $Y^{2}=Y_{\text {Spin }}$ and, while $Y_{S p i n}$ exists for both $S O\left(N_{c}\right)$ and $\operatorname{Spin}\left(N_{c}\right)$ gauge groups, the monopole $Y$ can be defined only for $S O\left(N_{c}\right)$. It is then necessary to further study these models from the perspective of their global properties.

In the analysis of the unitary theories there is a caveat, due to the presence of two different boundary conditions at the positions of the orientifolds. More concretely, the boundary condition corresponding to the $\mathrm{O}^{-}$at $x_{3}=$ $\pi R$ gives rise to a term in the superpotential that is not sensitive to the radius of the circle and can be sent to zero upon shrinking the radius of the circle. However, the other boundary condition $\mathrm{O}^{+}$at $x_{3}=0$ gives rise to a term in the superpotential that is sensitive to the radius of the circle. It should be interesting to elaborate further on this difference. 
Another interesting analysis that we did not perform consists in reproducing the dualities studied in Sec. V from the brane picture. In such cases there will be singular configurations, based on the presence of stacks of NS branes. These configurations were studied in [9] for the reduction of $4 \mathrm{~d}$ dualities to $3 \mathrm{~d}$. It should be possible to reproduce on such brane configurations the quadratic monopole superpotentials and the dualities discussed here in Sec. V.

Our analysis may also be generalized by considering $U\left(N_{c}\right)$ SQCD with two adjoints. The duality was derived in [7], inspired by the $4 \mathrm{~d}$ duality of [49]. It should be possible to see if a quadratic monopole deformation can be added to this duality and if it gives rise to a new IR duality. Other generalizations to the dualities with tensorial matter studied in [9] are expected as well.

We would like to conclude by observing a problem that appears in the unitary case with $N_{f}=N_{c}=1$. In this case the quadratic monopole deformation gives rise to a divergent partition function. This seems to be the case also when deforming the SQCDA/XYZ duality discussed in [50], by adding the deformations (5.3), (5.4) or (5.7). This corresponds to a flat direction in the Coulomb branch, and the partition function argument cannot be used in such cases. Further checks of the duality are necessary in this case.

\section{ACKNOWLEDGMENTS}

We thank Domenico Orlando, Sergio Benvenuti, and Susanne Reffert for valuable comments. The work of L. C. is supported in part by Vetenskapsrådet under Grant No. 20145517, by the STINT grant, and by the grant "Geometry and Physics" from the Knut and Alice Wallenberg Foundation.

\section{APPENDIX: THREE-SPHERE PARTITION FUNCTIONS}

In this Appendix we provide some useful formulas for the $3 \mathrm{~d} \mathcal{N}=2$ partition function on a squashed threesphere, used in the body of the paper. We refer to [51-54] for the original derivation in localization.

The partition function of a gauge theory is an integral over the Cartan of the gauge group. This is parametrized by the real scalars $\sigma$, representing the dynamical real scalar in the $\mathcal{N}=2$ vector multiplet. There are classical contributions, corresponding to the FI and to the CS terms in the action, and quantum contributions, represented by the oneloop determinants of the gauge and matter fields. These one-loop determinants can be formulated in terms of hyperbolic Gamma functions $\Gamma_{h}$ (see [31] for a definition and [55] for a physical interpretation),

$$
\begin{aligned}
\Gamma_{h}\left(z ; \omega_{1}, \omega_{2}\right) & \equiv \Gamma_{h}(z) \\
& \equiv \mathrm{e}^{\frac{\mathrm{i} \pi}{2 \omega_{1} \omega_{2}}\left((z-\omega)^{2}-\frac{\omega_{1}^{2}+\omega_{2}^{2}}{12}\right)} \prod_{\alpha=0}^{\infty} \frac{1-\mathrm{e}^{\frac{2 \pi i}{\omega_{1}}\left(\omega_{2}-z\right)} \mathrm{e}^{\frac{2 \pi i \omega_{2} \alpha}{\omega_{1}}}}{1-\mathrm{e}^{-\frac{2 \pi i}{\omega_{2}} z} \mathrm{e}^{-\frac{2 \pi \omega_{1} \alpha}{\omega_{2}}}},
\end{aligned}
$$

where the argument $z$ can be further refined by adding the contributions of the scalars in the background vector multiplets. The purely imaginary parameters $\omega_{1} \equiv \mathrm{i} b$ and $\omega_{2} \equiv \mathrm{i} b^{-1}$ are defined in terms of the real squashing parameter $b$ of the ellipsoid $S_{b}^{3}$, and $2 \omega \equiv \omega_{1}+\omega_{2}$.

There are two types of background symmetries, flavor and $R$-symmetries. We can turn on a collective background scalar $\mu$ for the first and $\Delta$ for the second, where $\Delta$ is the $R$-charge, equivalent to the mass dimension in three dimensions. We then define a holomorphic combination $\mu+\omega \Delta$. In other words, we can count the contribution of the $R$-symmetry by turning on an imaginary part for the real masses. We now restrict ourselves to the partition functions of interest in the paper, which are $U\left(N_{c}\right)$ SQCD with an adjoint and $U S p\left(2 N_{c}\right)$ SQCD with a traceless antisymmetric.

In the first case the partition function can be written as

$$
\begin{aligned}
& Z_{U\left(N_{c}\right)_{\kappa}}(\mu ; \nu ; \tau ; \eta) \\
& =\frac{1}{|W|} \int \prod_{i=1}^{N_{c}} \mathrm{~d} \sigma_{i} \mathrm{e}^{\mathrm{i} \pi \kappa \sigma_{i}^{2}+\mathrm{i} \pi \eta \sigma_{i}} \prod_{a=1}^{N_{f}} \Gamma_{h}\left(\mu_{a}+\sigma_{i}, \nu_{a}-\sigma_{i}\right) \\
& \quad \times \prod_{1 \leq i<j \leq N_{c}} \frac{\Gamma_{h}\left( \pm\left(\sigma_{i}-\sigma_{j}\right)+\tau\right)}{\Gamma_{h}\left( \pm\left(\sigma_{i}-\sigma_{j}\right)\right)}
\end{aligned}
$$

where we used the shorthand notations $\Gamma_{h}(x \pm y) \equiv$ $\Gamma_{h}(x+y) \Gamma_{h}(x-y)$ and $\Gamma_{h}(x, y)=\Gamma_{h}(x) \Gamma_{h}(y)$. The arguments in the lhs of (A2) refer, respectively, to the real masses of the fundamentals $(\mu)$, of the antifundamentals $(\nu)$, of the adjoint $(\tau)$ and of the FI $(\eta)$. Note that $|W|$ is the order of the Weyl group of $U\left(N_{c}\right)$. We also introduced a CS level $\kappa$ in (A2). When considering cases with vanishing CS, we omit the $\kappa$-dependence.

For $\operatorname{USp}\left(2 N_{c}\right)$ with an antisymmetric we have

$$
\begin{aligned}
& Z_{U S p\left(2 N_{c}\right)}(\mu ; \tau) \\
& =\frac{1}{|W|} \int \prod_{i=1}^{N_{c}} \mathrm{~d} \sigma_{i} \prod_{a=1}^{2 N_{f}} \Gamma_{h}\left( \pm \mu_{a}+\sigma_{i}\right) \\
& \quad \times \prod_{1 \leq i<j \leq N_{c}} \frac{\Gamma_{h}\left( \pm \sigma_{i} \pm \sigma_{j}+\tau\right)}{\left.\Gamma_{h}\left( \pm \sigma_{i} \pm \sigma_{j}\right)\right)} \prod_{i=1}^{N_{c}} \frac{1}{\Gamma_{h}\left( \pm 2 \sigma_{i}\right)},
\end{aligned}
$$

where the arguments in the lhs of (A3) refer, respectively, to the real masses of the fundamentals $(\mu)$ and of the antisymmetric $(\tau)$. In this case we omitted possible CS terms in (A3) because they do not play any relevant role in our discussion.

The real mass flows discussed on the field theory side correspond to the limit

$$
\lim _{x \rightarrow \infty} \Gamma_{h}(x)=\mathrm{e}^{\mathrm{i} \frac{\mathrm{i}}{2}(x-\omega)^{2}} .
$$


A real mass flow interpolating two dualities can be studied on the partition function by computing the limit (A4) on both sides of an identity between the partition functions of the dual phases. In order to reproduce the IR duality one has to focus on canceling the divergent contributions among the two sides of the identity.
[1] O. Aharony, S. S. Razamat, N. Seiberg, and B. Willett, $3 d$ dualities from $4 d$ dualities, J. High Energy Phys. 07 (2013) 149.

[2] V. Niarchos, Seiberg dualities and the $3 d / 4 d$ connection, J. High Energy Phys. 07 (2012) 075.

[3] O. Aharony, S. S. Razamat, N. Seiberg, and B. Willett, 3d dualities from $4 d$ dualities for orthogonal groups, J. High Energy Phys. 08 (2013) 099.

[4] K. Nii, $3 d$ duality with adjoint matter from $4 d$ duality, J. High Energy Phys. 02 (2015) 024.

[5] A. Amariti and C. Klare, A journey to $3 d$ : Exact relations for adjoint SQCD from dimensional reduction, J. High Energy Phys. 05 (2015) 148.

[6] A. Amariti, Integral identities for $3 d$ dualities with $\operatorname{SP}(2 \mathrm{~N})$ gauge groups, arXiv:1509.02199.

[7] C. Hwang, H. Kim, and J. Park, On $3 d$ Seiberg-like dualities with two adjoints, Fortschr. Phys. 66, 1800064 (2018).

[8] A. Amariti, D. Forcella, C. Klare, D. Orlando, and S. Reffert, The braneology of 3D dualities, J. Phys. A 48, 265401 (2015).

[9] A. Amariti, D. Forcella, C. Klare, D. Orlando, and S. Reffert, 4D/3D reduction of dualities: Mirrors on the circle, J. High Energy Phys. 10 (2015) 048.

[10] A. Amariti, D. Orlando, and S. Reffert, String theory and the 4D/3D reduction of Seiberg duality. A review, Phys. Rep. 705-706, 1 (2017).

[11] A. Amariti, D. Orlando, and S. Reffert, Monopole quivers and new 3D $N=2$ dualities, Nucl. Phys. B924, 153 (2017).

[12] A. Hanany and E. Witten, Type IIB superstrings, BPS monopoles, and three-dimensional gauge dynamics, Nucl. Phys. B492, 152 (1997).

[13] J. de Boer, K. Hori, Y. Oz, and Z. Yin, Branes and mirror symmetry in $N=2$ supersymmetric gauge theories in threedimensions, Nucl. Phys. B502, 107 (1997).

[14] N. M. Davies, T. J. Hollowood, V. V. Khoze, and M. P. Mattis, Gluino condensate and magnetic monopoles in supersymmetric gluodynamics, Nucl. Phys. B559, 123 (1999).

[15] N. M. Davies, T. J. Hollowood, and V. V. Khoze, Monopoles, affine algebras and the gluino condensate, J. Math. Phys. 44, 3640 (2003).

[16] T. Dimofte and D. Gaiotto, An E7 surprise, J. High Energy Phys. 10 (2012) 129.

[17] A. Amariti and L. Cassia, $U S p\left(2 N_{c}\right) \mathrm{SQCD}_{3}$ with antisymmetric: Dualities and symmetry enhancements, J. High Energy Phys. 02 (2019) 013.

[18] S. Benvenuti, A tale of exceptional $3 d$ dualities, J. High Energy Phys. 03 (2019) 125.

[19] M. Fazzi, A. Lanir, S. S. Razamat, and O. Sela, Chiral $3 d \mathrm{SU}(3) \mathrm{SQCD}$ and $\mathcal{N}=2$ mirror duality, J. High Energy Phys. 11 (2018) 025.
[20] S. Benvenuti and S. Pasquetti, $3 d \mathcal{N}=2$ mirror symmetry, pq-webs and monopole superpotentials, J. High Energy Phys. 08 (2016) 136.

[21] F. Benini, S. Benvenuti, and S. Pasquetti, SUSY monopole potentials in $2+1$ dimensions, J. High Energy Phys. 08 (2017) 086.

[22] S. Benvenuti and S. Giacomelli, Supersymmetric Gauge Theories with Decoupled Operators and Chiral Ring Stability, Phys. Rev. Lett. 119, 251601 (2017).

[23] S. Benvenuti and S. Giacomelli, Abelianization and sequential confinement in $2+1$ dimensions, J. High Energy Phys. 10 (2017) 173.

[24] N. Aghaei, A. Amariti, and Y. Sekiguchi, Notes on integral identities for $3 d$ supersymmetric dualities, J. High Energy Phys. 04 (2018) 022.

[25] P. Agarwal, On dimensional reduction of $4 d \quad N=1$ Lagrangians for Argyres-Douglas theories, J. High Energy Phys. 03 (2019) 011.

[26] K. Maruyoshi and J. Song, Enhancement of Supersymmetry via Renormalization Group Flow and the Superconformal Index, Phys. Rev. Lett. 118, 151602 (2017).

[27] K. Maruyoshi and J. Song, $\mathcal{N}=1$ deformations and RG flows of $\mathcal{N}=2$ SCFTs, J. High Energy Phys. 02 (2017) 075.

[28] P. Agarwal, K. Maruyoshi, and J. Song, $\mathcal{N}=1$ Deformations and $\mathrm{RG}$ flows of $\mathcal{N}=2$ SCFTs, part II: Non-principal deformations, J. High Energy Phys. 12 (2016) 103; Addendum 04 (2017) 113.

[29] P. Agarwal, A. Sciarappa, and J. Song, $\mathcal{N}=1$ Lagrangians for generalized Argyres-Douglas theories, J. High Energy Phys. 10 (2017) 211.

[30] S. Benvenuti and S. Giacomelli, Lagrangians for generalized Argyres-Douglas theories, J. High Energy Phys. 10 (2017) 106.

[31] F. van de Bult, Hyperbolic hypergeometric functions, Thesis, 2007, http://math.caltech.edu/ vdbult/Thesis.pdf.

[32] A. Collinucci, S. Giacomelli, R. Savelli, and R. Valandro, T-branes through $3 d$ mirror symmetry, J. High Energy Phys. 07 (2016) 093.

[33] A. Collinucci, S. Giacomelli, and R. Valandro, T-branes, monopoles and S-duality, J. High Energy Phys. 10 (2017) 113.

[34] S. Giacomelli and N. Mekareeya, Mirror theories of $3 d \mathcal{N}=2$ SQCD, J. High Energy Phys. 03 (2018) 126.

[35] A. Amariti, SUSY breaking in monopole quivers, arXiv:1808.09983.

[36] F. Aprile, S. Pasquetti, and Y. Zenkevich, Flipping the head of $\mathrm{T}[\mathrm{SU}(\mathrm{N})]$ : Mirror symmetry, spectral duality and monopoles, J. High Energy Phys. 04 (2019) 138.

[37] A. Amariti, I. Garozzo, and N. Mekareeya, New $3 d \mathcal{N}=2$ dualities from quadratic monopoles, J. High Energy Phys. 11 (2018) 135. 
[38] O. Aharony, IR duality in $d=3 N=2$ supersymmetric $U S p\left(2 N_{c}\right)$ and $U\left(N_{c}\right)$ gauge theories, Phys. Lett. B 404, 71 (1997).

[39] H. Kim and J. Park, Aharony dualities for $3 d$ theories with adjoint matter, J. High Energy Phys. 06 (2013) 106.

[40] S. Kim, K.-M. Lee, H.-U. Yee, and P. Yi, The $N=1 *$ theories on $\mathbb{R}^{(1+2)} \times S^{1}$ with twisted boundary conditions, J. High Energy Phys. 08 (2004) 040.

[41] H. Garland and M. K. Murray, Kac-Moody monopoles and periodic instantons, Commun. Math. Phys. 120, 335 (1988).

[42] W. Nahm, The construction of all self-dual multimonopoles by the ADHM method, in Monopoles in Quantum Field Theory, Trieste, Italy, 1981 (1982), pp. 87-94.

[43] D. J. Gross, R. D. Pisarski, and L. G. Yaffe, QCD and instantons at finite temperature, Rev. Mod. Phys. 53, 43 (1981).

[44] K.-M. Lee and P. Yi, Monopoles and instantons on partially compactified D-branes, Phys. Rev. D 56, 3711 (1997).

[45] T. C. Kraan and P. van Baal, Exact T duality between calorons and Taub-NUT spaces, Phys. Lett. B 428, 268 (1998).

[46] K.-M. Lee, Instantons and magnetic monopoles on $\mathbb{R}^{3} \times S^{1}$ with arbitrary simple gauge groups, Phys. Lett. B 426, 323 (1998).
[47] A. Hanany and J. Troost, Orientifold planes, affine algebras and magnetic monopoles, J. High Energy Phys. 08 (2001) 021.

[48] S. Cremonesi, G. Ferlito, A. Hanany, and N. Mekareeya, Coulomb branch and the moduli space of instantons, J. High Energy Phys. 12 (2014) 103.

[49] J. H. Brodie, Duality in supersymmetric $\mathrm{SU}\left(N_{c}\right)$ gauge theory with two adjoint chiral superfields, Nucl. Phys. B478, 123 (1996).

[50] F. Nieri, Y. Pan, and M. Zabzine, $3 d$ mirror symmetry from S-duality, Phys. Rev. D 98, 126002 (2018).

[51] A. Kapustin, B. Willett, and I. Yaakov, Exact results for Wilson loops in superconformal Chern-Simons theories with matter, J. High Energy Phys. 03 (2010) 089.

[52] D. L. Jafferis, The exact superconformal R-symmetry extremizes Z, J. High Energy Phys. 05 (2012) 159.

[53] N. Hama, K. Hosomichi, and S. Lee, Notes on SUSY gauge theories on three-sphere, J. High Energy Phys. 03 (2011) 127.

[54] N. Hama, K. Hosomichi, and S. Lee, SUSY gauge theories on squashed three-spheres, J. High Energy Phys. 05 (2011) 014.

[55] F. Benini, C. Closset, and S. Cremonesi, Comments on $3 d$ Seiberg-like dualities, J. High Energy Phys. 10 (2011) 075. 